\title{
Constraints Optimal Control Governing by Triple Nonlinear Hyperbolic Boundary Value Problem
}

\author{
Jamil A. Ali Al-Hawasy (i) and Lamyaa H. Ali \\ Department of Mathematics, College of Science, Mustansiriyah University, Baghdad, Iraq \\ Correspondence should be addressed to Jamil A. Ali Al-Hawasy; jhawassy17@uomustansiriyah.edu.iq
}

Received 27 October 2019; Accepted 28 January 2020; Published 9 April 2020

Academic Editor: Dogan Kaya

Copyright (c) 2020 Jamil A. Ali Al-Hawasy and Lamyaa H. Ali. This is an open access article distributed under the Creative Commons Attribution License, which permits unrestricted use, distribution, and reproduction in any medium, provided the original work is properly cited.

\begin{abstract}
The focus of this work lies on proving the existence theorem of a unique state vector solution (Stvs) of the triple nonlinear hyperbolic boundary value problem (TNHBVP) when the classical continuous control vector (CCCVE) is fixed by using the Galerkin method (Galm), proving the existence theorem of a unique constraints classical continuous optimal control vector (CCCOCVE) with vector state constraints (equality EQVC and inequality INEQVC). Also, it consists of studying for the existence and uniqueness adjoint vector solution (Advs) of the triple adjoint vector equations (TAEqs) associated with the considered triple state equations (Tsteqs). The Fréchet Derivative (Frde.) of the Hamiltonian (HAM) is found. At the end, the theorems for the necessary conditions and the sufficient conditions of optimality (Necoop and Sucoop) are achieved.
\end{abstract}

\section{Introduction}

The subject of optimal control problem (OCP) plays a basic role in many real life problems in different branches of sciences; for example, in, medicine [1], engineering and social sciences [2], biology [3], ecology [4], electric power [5], aerospace [6], and many other branches.

This role encouraged many researchers to go deeply into studying the OCPS governed by differential equations (deqs). Such OCP problems are studied at the beginning for the systems which are controlled by nonlinear ordinary deqs (nodeqs) [7] or by linear deqs (lpdeqs) [8]. Later great interests have been made to study this subject but for systems which are controlled by pdeqs of elliptic type (ET) [9], or of hyperbolic type (HT) [10], or of parabolic type (PT) [11], or by couple of npdeqs of ET [12], or of PT [13], or of HT [14].

Recently, the attention in this subject is magnified to deal with more general types as the studying the CCCOPCVE controlling by TBVP of ET [15], and of PT [16]. All these studies motivated us to look deep inside the CCCOPCVE controlled by TNHBVP.

In this work and at first, we give a mathematical description for the CCCOPCVE, and then the TNHBVP is written in its weak form (wkf), and the existence and uniqueness theorem of the Stvs for the TNHBVP using the Galm with the Aubin compactness theorem is proved under appropriate hypotheses when the CCCVE is given. Under reasonable hypotheses, the objective function and the EQVC and INEQVC are proved continuous. The proof of the existence theorem of a CCCOPCVE governed by the TNHBVP is achieved. Under a certain hypotheses, the study of the existence theorem for a unique Advs of the TAEqs associated with the considered Tsteqs is done. The Fréchet Derivative (Frde.) of the HAM is found. Finally, the Necoop and the Sucoop theorems for the CCCOPCVE are proved.

1.1. Problem Description. Let $I=[0, T], T<\infty, E$ be a bounded and open region in $\mathbb{R}^{2}$ with Lipschitz (Lip.) boundary $\partial E, \Pi=E \times I$, and $\partial \Pi=\partial E \times I$. The considered CCOPCP consists of the Steq which is given by the TNLHPDEqs:

$$
\begin{array}{ll}
\psi_{1 t t}-\Delta \psi_{1}+\psi_{1}-\psi_{2}-\psi_{3}=k_{1}\left(x, t, \psi_{1}, \omega_{1}\right), & \text { in } \Pi, \\
\psi_{2 t t}-\Delta \psi_{2}+\psi_{2}+\psi_{3}+\psi_{1}=k_{2}\left(x, t, \psi_{2}, \omega_{2}\right), & \text { in } \Pi, \\
\psi_{3 t t}-\Delta \psi_{3}+\psi_{3}+\psi_{1}-\psi_{2}=k_{3}\left(x, t, \psi_{3}, \omega_{3}\right), & \text { in } \Pi,
\end{array}
$$

with the BCs and ICs. 


$$
\begin{gathered}
\psi_{1}(x, t)=0, \psi_{2}(x, t)=0, \psi_{3}(x, t)=0, \quad \text { on } \partial \Pi, \\
\psi_{1}(x, 0)=\psi_{1}^{0}(x), \psi_{2}(x, 0)=\psi_{2}^{0}(x), \psi_{3}(x, 0)=\psi_{3}^{0}(x), \quad \text { on } E, \\
\psi_{1 t}(x, 0)=\psi_{1}^{1}(x), \psi_{1 t}(x, 0)=\psi_{2}^{1}(x), \psi_{3 t}(x, 0)=\psi_{3}^{1}(x), \quad \text { on } E,
\end{gathered}
$$

where $\vec{\psi}=\left(\psi_{1}, \psi_{2}, \psi_{3}\right) \in\left(H^{1}(E)\right)^{3}$ is the Stvs, corresponding to the CCCVE $\vec{\omega}=\left(\omega_{1}, \omega_{2}, \omega_{3}\right) \in\left(L^{2}(\Pi)\right)^{3}$ and $\left(k_{1}, k_{2}, k_{3}\right) \in\left(L^{2}(\Pi)\right)^{3}$ is a function defined on $(\Pi \times \mathscr{R} \times$ $\left.C_{1}\right) \times\left(\Pi \times \mathscr{R} \times \complement_{2}\right) \times\left(\Pi \times \mathscr{R} \times \complement_{3}\right)$ with $\complement_{i} \subset \mathscr{R}$, for $i=1,2,3$.

The controls set are $\vec{\omega} \in \overrightarrow{\widehat{W}}, \overrightarrow{\widehat{W}} \subset\left(L^{2}(\Pi)\right)^{3}$ with $\vec{W}=\left\{\vec{W} \in\left(L^{2}(\Pi)\right)^{3} \mid \vec{W} \in \vec{C}\right.$, a.e. in $\left.\Pi\right\}$, with $\vec{C} \subset \mathscr{R}^{3}$.

The cost function is

$$
M_{0}(\vec{\omega})=\sum_{i=1}^{3} \int_{\Pi} m_{0 i}\left(x, t, \psi_{i}, \omega_{i}\right) \mathrm{d} x \mathrm{~d} t
$$

The EQVC and INEQVC on the state vectors are

$$
\begin{array}{r}
M_{r}(\vec{\omega})=\sum_{i=1}^{3} \int_{\Pi} m_{r i}\left(x, t, \psi_{i}, \omega_{i}\right) \mathrm{d} x \mathrm{~d} t=0, \\
1 \leq r \leq p, \\
M_{r}(\vec{\omega})=\sum_{i=1}^{3} \int_{\Pi} m_{r i}\left(x, t, \psi_{i}, \omega_{i}\right) \mathrm{d} x \mathrm{~d} t \leq 0, \\
p+1 \leq r \leq q .
\end{array}
$$

The set of admissible control vector is $\vec{W}_{A}=$ $\left\{\vec{\omega} \in \vec{W} \mid M_{r}(\vec{\omega})=0, M_{r+p}(\vec{\omega}) \leq 0,1 \leq r \leq p\right\}$.

The continuous optimal control problem is to find $\vec{\omega} \in \overrightarrow{\widehat{W}}_{A}$ such that $M_{0}(\vec{\omega})=\overrightarrow{\widehat{w}} \in \overrightarrow{\widehat{W}}_{A}^{\text {min }} M_{0}(\overrightarrow{\widehat{w}})$.

Let $\vec{\Upsilon}=. \Upsilon_{1} \times . \Upsilon_{2} \times \Upsilon_{3}=\left\{\vec{v}: \vec{v} \in\left(H^{1}(\Omega)\right)^{3}\right.$, with $v_{1}=v_{2}=v_{2}=0$ on $\left.\partial E\right\}, \vec{v}=\left(v_{1}, v_{2}, v_{3}\right)$.

We denote by $(v, v),(v, v)_{1}$, and $(\vec{v}, \vec{v})_{1}=\sum_{i=1}^{3}\left(v_{i}, v_{i}\right)_{1}$ to the inner products in $L^{2}(E), H^{1}(E)$, and $\vec{\Upsilon}$ respectively while the norms in these spaces is denoted by $v_{0}, v_{1}$, and $\vec{v}_{1}^{2}=\sum_{i=1}^{3} v_{i 1}^{2}, \vec{\Upsilon}^{*}$ is denoted the dual of $\vec{\Upsilon}$. Also, the symbol-will be used to indicate that the convergence of a sequence is weak, while the strong convergence of a sequence will be indicated by $\longrightarrow$.

The wkf of problem (1)-(6) when $\vec{\psi} \in\left(H_{0}^{1}(E)\right)^{3}$ is given almost everywhere on $I$ for each $v_{1} \in \Upsilon_{1}, v_{2} \in \Upsilon_{2}$, and $v_{3} \in \Upsilon_{3}$ :

$$
\begin{aligned}
\left\langle\psi_{1 t t}, v_{1}\right\rangle+\left(\nabla \psi_{1}, \nabla v_{1}\right)+\left(\psi_{1}-\psi_{2}-\psi_{3}, v_{1}\right) & =\left(k_{1}\left(\psi_{1}, \omega_{1}\right), v_{1}\right), \quad \psi_{1}(\cdot, t) \in Y_{1}, \\
\left(\psi_{1}^{0}, v_{1}\right) & =\left(\psi_{1}(0), v_{1}\right), \\
\left(\psi_{1}^{1}, v_{1}\right) & =\left(\psi_{1 t}(0), v_{1}\right), \\
\left\langle\psi_{2 t t}, v_{2}\right\rangle+\left(\nabla \psi_{2}, \nabla v_{2}\right)+\left(\psi_{2}+\psi_{1}+\psi_{3}, v_{2}\right) & =\left(k_{2}\left(\psi_{2}, \omega_{2}\right), v_{2}\right), \quad \psi_{2}(\cdot, t) \in Y_{2}, \\
\left(\psi_{2}^{0}, v_{2}\right) & =\left(\psi_{2}(0), v_{2}\right), \\
\left(\psi_{2}^{1}, v_{2}\right) & =\left(\psi_{2 t}(0), v_{2}\right), \\
\left\langle\psi_{3 t t}, v_{3}\right\rangle+\left(\nabla \psi_{3}, \nabla v_{3}\right)+\left(\psi_{3}+\psi_{1}-\psi_{2}, v_{3}\right) & =\left(k_{3}\left(\psi_{3}, \omega_{3}\right), v_{3}\right), \quad \psi_{3}(\cdot, t) \in Y_{3}, \\
\left(\psi_{3}^{0}, v_{3}\right) & =\left(\psi_{3}(0), v_{3}\right), \\
\left(\psi_{3}^{1}, v_{3}\right) & =\left(\psi_{3 t}(0), v_{3}\right) .
\end{aligned}
$$

The following assumptions (Assums.) are needed to investigate the classical continuous optimal control problem (CCOPCP).
Assumption A. $k_{i}$ is of the Carathéodory type (Caraty.) on $\Pi \times\left(\mathscr{R} \times \complement_{i}\right)$ and satisfies the following conditions for $(x, t) \in \Pi$ and $\forall i=1,2,3$ : 


$$
\begin{aligned}
\left|k_{i}\left(x, t, \psi_{i}, \omega_{i}\right)\right| & \leq F_{i}(x, t)+\beta_{i}\left|\psi_{i}\right|, \quad \text { where } \psi_{i}, \omega_{i} \in \mathscr{R}, \beta_{i}>0, F_{i} \in L^{2}(\Pi), \\
\left|k_{i}\left(x, t, \psi_{i}, \omega_{i}\right)-k_{i}\left(x, t, \bar{\psi}_{i}, \omega_{i}\right)\right| & \leq L_{i}\left|\psi_{i}-\bar{\psi}\right|, \quad \text { where } \psi_{i}, \bar{\psi}_{i}, \omega_{i} \in \mathscr{R}, L_{i}>0 .
\end{aligned}
$$

1.2. The Solution of the State Equations. In this part, the existence theorem of a unique solution for triple nonlinear hyperbolic partial differential equations (TNLHPDEQs) under Assumption A is proved when the control vector is given, and the following proposition will be needed.

Proposition 1 (see [17]). Suppose $D \subset \mathscr{R}^{s}(s=2,3), k: D \times$ $\mathscr{R}^{n} \longrightarrow \mathscr{R}^{m}$ is of Caraty. It satisfies $\|k(v, x)\| \leq \alpha(v)+\beta(v)\|x\|^{a}$, for each $(v, x) \in D \times \mathscr{R}^{n}$, where $x \in L^{b}\left(D, \mathscr{R}^{n}\right), \alpha \in L^{1}(D, R), \beta \in L^{(b / b-a)}(D, R), a \in[0, b]$, if $b \neq \infty, \alpha=0$ if $b=\infty$. Then, the functional $K(x)=\int_{D} k(v, x(v)) d v$ is cont.

Theorem 1. Existence and Uniqueness of the Stvs: with Assumption A, for any given $\vec{\omega} \in\left(L^{2}(\Pi)\right)^{3}$, the wkf of (10)-(15) has a unique solution $\vec{\psi}=\left(\psi_{1}, \psi_{2}, \psi_{3}\right)$ s.t. $\vec{\psi} \in\left(L^{2}(I, \Upsilon)\right)^{3}, \quad \vec{\psi}_{t}=\left(\psi_{1 t}, \psi_{2 t}, \psi_{3 t}\right) \in\left(L^{2}(\Pi)\right)^{3}, \quad$ and $\vec{\psi}_{t t}=\left(\psi_{1 t}, \psi_{2 t}, \psi_{3 t}\right) \in\left(L^{2}\left(I, \Upsilon^{*}\right)\right)^{3}$.

Proof. Let $\vec{\Upsilon}_{n}=\Upsilon_{n} \times \Upsilon_{n} \times \Upsilon_{n} \subset \vec{\Upsilon}$ (for each $n$ ) be the set of cont. and piecewise affine function in $E$. $\left\{\vec{\Upsilon}_{n}\right\}_{n=1}^{\infty}$ be a sequence of subspaces of $\vec{\Upsilon}$, such that $\forall \vec{v}=\left(v_{1}, v_{2}\right.$, $\left.v_{3}\right) \in \vec{\Upsilon}$, there exists a sequence $\left\{\vec{v}_{n}\right\}$ with $\vec{v}_{n}=\left(v_{1 n}, v_{2 n}\right.$, $\left.v_{3 n}\right) \in \vec{\Upsilon}_{n}, \forall n$, and $\vec{v}_{n} \longrightarrow \vec{v}$ in $\vec{Y} \Longrightarrow \vec{v}_{n} \Longrightarrow \vec{v}$ in $\left(L^{2}(E)\right)^{3} \cdot\left\{\vec{v}_{j}=\left(v_{1 j}, v_{2 j}, v_{3 j}\right): j=1,2, \ldots, n\right\}$ be a finite basis of $\vec{\Upsilon}_{n}$ (where $\vec{v}_{j}$ is cont. function in $E$, with $\vec{v}_{j}(x)=0$ on the boundary $\partial E)$ and let $\vec{\psi}_{n}=\left(\psi_{1 n}, \psi_{2 n}, \psi_{3 n}\right)$ be the Galerkin approximate solution (Galso) to the exact solution $\vec{\psi}=\left(\psi_{1}, \psi_{2}, \psi_{3}\right)$ such that

$$
\psi_{i n}=\sum_{j=1}^{n} c_{i j}(t) v_{i j}(x),
$$

where $c_{i j}(t)$ are unknown functions of $t, \forall j=1,2, \ldots, n$, and. $\forall i=1,2,3$.

The wkf of (10)-(15) is approximated with respect to $x$ using the Galm, and substituting $\psi_{\text {int }}=\zeta_{\text {in }}(i=1,2,3)$ in the obtained equations, they become for each $v_{1}, v_{2}, v_{3} \in \Upsilon_{n}$ :

$$
\begin{aligned}
\left\langle\zeta_{1 n t}, v_{1}\right\rangle+\left(\nabla \psi_{1 n}, \nabla v_{1}\right)+\left(\psi_{1 n}, v_{1}\right)-\left(\psi_{2 n}, v_{1}\right)-\left(\psi_{3 n}, v_{1}\right) & =\left(k_{1}\left(\psi_{1 n}, \omega_{1}\right), v_{1}\right), \\
\left(\psi_{1 n}^{0}, v_{1}\right) & =\left(\psi_{1}^{0}, v_{1}\right), \\
\left(\psi_{1 n}^{1}, v_{1}\right) & =\left(\psi_{1}^{1}, v_{1}\right), \\
\left\langle\zeta_{2 n t}, v\right\rangle+\left(\nabla \psi_{2 n}, \nabla v_{2}\right)+\left(\psi_{2 n}, v_{2}\right)+\left(\psi_{3 n}, v_{2}\right)+\left(\psi_{1 n}, v_{2}\right) & =\left(k_{2}\left(\psi_{2 n}, \omega_{2}\right), v_{2}\right), \\
\left(\psi_{2 n}^{0}, v_{2}\right) & =\left(\psi_{2}^{0}, v_{2}\right), \\
\left(\psi_{2 n}^{1}, v_{2}\right) & =\left(\psi_{2}^{1}, v_{2}\right), \\
\left\langle\zeta_{3 n t}, v_{3}\right\rangle+\left(\nabla \psi_{3 n}, \nabla v_{3}\right)+\left(\psi_{3 n}, v_{3}\right)+\left(\psi_{1 n}, v_{3}\right)-\left(\psi_{2 n}, v_{3}\right) & =\left(k_{3}\left(\psi_{3 n}, \omega_{3}\right), v_{3}\right), \\
\left(\psi_{3 n}^{0}, v_{3}\right) & =\left(\psi_{3}^{0}, v_{3}\right), \\
\left(\psi_{3 n}^{1}, v_{3}\right) & =\left(\psi_{3}^{1}, v_{3}\right),
\end{aligned}
$$

where $\quad \psi_{\text {in }}^{0}=\psi_{\text {in }}^{0}(x)=\psi_{\text {in }}(x, 0) \in \Upsilon_{n} \quad$ (respectively, $\left.\zeta_{\text {in }}^{0}=\psi_{\text {in }}^{1}=\psi_{\text {in }}^{1}(x)=\psi_{\text {int }}(x, 0) \in L^{2}(E)\right)$ be the projection of $y_{i}^{0}$ onto $V$ (be the projection of $\psi_{i}^{1}=\psi_{i t}$ onto $L^{2}(E)$ ), $\forall i=1,2,3$, i.e.,

$$
\begin{aligned}
& \psi_{\text {in }}^{0} \longrightarrow \psi_{i}^{0} \text { in } V, \quad \text { with }\left\|\vec{\psi}_{n}^{0}\right\|_{1} \leq b_{0} \text { and }\left\|\vec{\psi}_{n}^{0}\right\|_{0} \leq b_{0}, \\
& \psi_{\text {in }}^{1} \longrightarrow \psi_{i}^{1}, \quad \text { in } L^{2}(E) \text { and }\left\|\vec{\psi}_{n}^{1}\right\|_{0} \leq b_{1} .
\end{aligned}
$$

Substituting (17) for each $i=1,2,3$, respectively, in the pairs (18) and (19), (20) and (21), and in (22) and (23), setting $v_{1}=v_{1 i}, v_{2}=v_{2 i}, v_{3}=v_{3 i}$, the obtained equations will be written as the following $1^{\text {st }}$ order nodeqs with their ICs and have a unique solution $\vec{\psi}_{n} \in C(I, \vec{\Upsilon})$, i.e., for each $l=$ $1,2,3$ and $\bar{m}=0,1$ : 


$$
\begin{aligned}
A_{1} D_{1}^{\prime}(t)+B_{1} C_{1}(t)-E_{1} C_{2}(t)-H_{1} C_{3}(t) & =b_{1}\left(\bar{Y}_{1}^{T}(x) C_{1}(t)\right), \\
A_{1} C_{1}(0) & =b_{1}^{0}, \\
A_{1} D_{1}(0) & =b_{1}^{1}, \\
A_{2} D_{2}^{\prime}(t)+B_{2} C_{2}(t)+E_{2} C_{1}(t)+H_{2} C_{3}(t) & =b_{2}\left(\bar{Y}_{2}^{T}(x) C_{2}(t)\right), \\
A_{2} C_{2}(0) & =b_{2}^{0}, \\
A_{2} D_{2}(0) & =b_{2}^{1}, \\
A_{3} D_{3}^{\prime}(t)+B_{3} C_{3}(t)+E_{3} C_{1}(t)-H_{3} C_{3}(t) & =b_{3}\left(\bar{Y}_{3}^{T}(x) C_{3}(t)\right), \\
A_{3} C_{3}(0) & =b_{3}^{0}, \\
A_{3} D_{3}(0) & =b_{3}^{1},
\end{aligned}
$$

where $\quad C_{l}(t)=\left(c_{l j}(t)\right)_{n \times 1}, \quad C_{2}^{\prime}(t)=\left(c_{2 j}^{\prime}(t)\right)_{n \times 1}, \quad D_{l}^{\prime}(t)=$ $\left(d_{l j}^{\prime}(t)\right)_{n \times 1}, \quad D_{l}(t)=\left(d_{l j}(t)\right)_{n \times 1}, \quad b_{l}=\left(b_{l i}\right)_{n \times 1}, \quad b_{l i}=\left(k_{l}\left(V_{l}^{T}\right.\right.$ $\left.\left.c_{l}(t), \omega_{l}\right), v_{l i}\right), \quad b_{l}^{\bar{m}}=\left(b_{l j}^{\bar{m}}\right), \quad b_{l j}^{\bar{m}}=\left(\psi_{l}^{\bar{m}}, v_{l j}\right), \quad A_{l}=\left(a_{l i j}\right)_{n \times n}$, $a_{l i j}=\left(v_{l j}, v_{l i}\right), E_{1}=\left(e_{1 i j}\right)_{n \times n}, e_{1 i j}=\left(v_{2 j}, v_{1 i}\right), H_{1}=\left(f_{1 i j}\right)_{n \times n}$ $f_{1 i j}=\left(v_{3 j}, v_{1 i}\right), \quad E_{2}=\left(e_{2 i j}\right)_{n \times n}, \quad e_{2 i j}=\left(v_{1 j}, v_{2 i}\right), \quad H_{2}=$ $\left(f_{2 i j}\right)_{n \times n}, \quad f_{2 i j}=\left(v_{3 j}, v_{2 i}\right), E_{3}=\left(e_{3 i j}\right)_{n \times n}, e_{3 i j}=\left(v_{1 j}, v_{3 i}\right)$,
$H_{3}=\left(f_{3 i j}\right)_{n \times n}, \quad f_{3 i j}=\left(v_{2 j}, v_{3 i}\right), \quad B_{l}=\left(b_{l i j}\right)_{n \times n}$, and $b_{l i j}=$ $\left[\left(\nabla v_{l j}, \nabla v_{l i}\right)+\left(v_{l j}, v_{l i}\right)\right]$.

Then, corresponding to the sequence $\left\{\vec{\Upsilon}_{n}\right\}$, there exists a sequence of the following approximation problems, i.e., for each $\vec{v}_{n}=\left(v_{1 n}, v_{2 n}, v_{3 n}\right) \subset \vec{\Upsilon}_{n}$, and $n=1,2, \ldots$ :

$$
\begin{aligned}
\left\langle\psi_{1 n t t}, v_{1 n}\right\rangle+\left(\nabla \psi_{1 n}, \nabla v_{1 n}\right)+\left(\psi_{1 n}-\psi_{2 n}-\psi_{3 n}, v_{1 n}\right) & =\left(k_{1}\left(\psi_{1 n}, \omega_{1}\right), v_{1 n}\right), \\
\left(\psi_{1 n}^{0}, v_{1 n}\right) & =\left(\psi_{1}^{0}, v_{1 n}\right), \\
\left(\psi_{1 n}^{1}, v_{1}\right) & =\left(\psi_{1}^{1}, v_{1 n}\right), \\
\left\langle\psi_{2 n t t}, v_{2 n}\right\rangle+\left(\nabla \psi_{2 n}, \nabla v_{2 n}\right)+\left(\psi_{2 n}+\psi_{3 n}+\psi_{1 n}, v_{2 n}\right) & =\left(k_{2}\left(\psi_{2 n}, \omega_{2}\right), v_{2 n}\right), \\
\left(\psi_{2 n}^{0}, v_{2 n}\right) & =\left(\psi_{2}^{0}, v_{2 n}\right), \\
\left(\psi_{2 n}^{1}, v_{2}\right) & =\left(\psi_{2}^{1}, v_{2 n}\right), \\
\left\langle\psi_{3 n t t}, v_{3 n}\right\rangle+\left(\nabla \psi_{3 n}, \nabla v_{3 n}\right)+\left(\psi_{3 n}+\psi_{1 n}, v_{3 n}\right) & =\left(k_{3}\left(\psi_{3 n}, \omega_{3}\right), v_{3 n}\right), \\
\left(\psi_{3 n}^{0}, v_{3 n}\right) & =\left(\psi_{3}^{0}, v_{3 n}\right), \\
\left(\psi_{3 n}^{1}, v_{3}\right) & =\left(\psi_{3}^{1}, v_{3 n}\right),
\end{aligned}
$$

which has a sequence of unique solution $\left\{\vec{\psi}_{n}\right\}$. Substituting $v_{\text {in }}=\psi_{\text {int }}$ for $i=1,2,3$ in (25)-(27), respectively, adding the three obtained equations together, and employing Lemma
1.2 in [18] for the first term of the LHS, to get (33) which is given by

$$
\begin{aligned}
\frac{\mathrm{d}}{\mathrm{d} t}\left[\left\|\vec{\psi}_{n t}(t)\right\|_{0}^{2}+\left\|\vec{\psi}_{n}\right\|_{1}^{2}\right]= & 2\left(\left(\psi_{2 n}, \psi_{1 n t}\right)+\left(\psi_{3 n}, \psi_{1 n t}\right)-\left(\psi_{1 n}, \psi_{2 n t}\right)-\left(\psi_{3 n}, \psi_{2 n t}\right)-\left(\psi_{1 n}, \psi_{3 n t}\right)+\left(\psi_{2 n}, \psi_{3 n t}\right)\right. \\
& \left.+\left(k_{1}\left(\psi_{1 n}, \omega_{1}\right), \psi_{1 n t}\right)+\left(k_{2}\left(\psi_{2 n}, \omega_{2}\right), \psi_{2 n t}\right)+\left(k_{3}\left(\psi_{3 n}, \omega_{3}\right), \psi_{3 n t}\right)\right) .
\end{aligned}
$$

Or (33) can be rewritten as (34) which is

$$
\begin{aligned}
\frac{\mathrm{d}}{\mathrm{d} t}\left[\left\|\vec{\psi}_{n t}(t)\right\|_{0}^{2}+\left\|\vec{\psi}_{n}\right\|_{1}^{2}\right] \leq 2\left(\left|\left(\psi_{2 n}, \psi_{1 n t}\right)\right|+\left|\left(\psi_{3 n}, \psi_{1 n t}\right)\right|+\left|\left(\psi_{1 n}, \psi_{2 n t}\right)\right|+\left|\left(\psi_{3 n}, \psi_{2 n t}\right)\right|+\left|\left(\psi_{1 n}, \psi_{3 n t}\right)\right|\right. \\
\left.\quad+\left|\left(\psi_{2 n}, \psi_{2 n t}\right)\right|+\left|\left(k_{1}\left(\psi_{1 n}, \omega_{1}\right), \psi_{1 n t}\right)\right|+\left|\left(k_{2}\left(\psi_{2 n}, \omega_{2}\right), \psi_{2 n t}\right)\right|+\left|\left(k_{3}\left(\psi_{3 n}, \omega_{3}\right), \psi_{3 n t}\right)\right|\right) .
\end{aligned}
$$


Using Assumption A for the RHS of (34), integrating both sides on $[0, t]$, using $\left\|\psi_{\text {in }}\right\|_{0} \leq\left\|\psi_{\text {in }}\right\|_{1} \leq\left\|\vec{\psi}_{n}\right\|_{1}$, and $\left\|\psi_{\text {int }}\right\|_{0} \leq\left\|\vec{\psi}_{n t}\right\|_{0}$, we get

$$
\begin{aligned}
& \int_{0}^{t} \frac{\mathrm{d}}{\mathrm{d} t}\left[\left\|\vec{\psi}_{n t}(t)\right\|_{0}^{2}+\left\|\vec{\psi}_{n}\right\|_{1}^{2}\right] \mathrm{d} t \\
& \leq \int_{0}^{t}\left(\left\|\vec{\psi}_{n t}\right\|_{0}^{2}+\left\|\vec{\psi}_{n}\right\|_{1}^{2}\right) \mathrm{d} t+\int_{0}^{t} \sum_{i=1}^{3}\left\|F_{i}\right\|_{0}^{2} \mathrm{~d} t \\
& \quad+\beta_{4} \int_{0}^{t}\left(\left\|\vec{\psi}_{n t}\right\|_{0}^{2}+\left\|\vec{\psi}_{n}\right\|_{1}^{2}\right) \mathrm{d} t+\int_{0}^{t}\left\|\vec{\psi}_{n t}\right\|_{0}^{2} \mathrm{~d} t \\
& \leq \sum_{i=1}^{3}\left\|F_{i}\right\|_{Q}^{2}+\beta_{6} \int_{0}^{t}\left(\left\|\vec{\psi}_{n t}\right\|_{0}^{2}+\left\|\vec{\psi}_{n}\right\|_{1}^{2}\right) \mathrm{d} t \\
& \leq \beta_{8}+\beta_{7} \int_{0}^{t}\left(\left\|\vec{\psi}_{n t}\right\|_{0}^{2}+\left\|\vec{\psi}_{n}\right\|_{1}^{2}\right) \mathrm{d} t,
\end{aligned}
$$

where $\beta_{4}=\sum_{i=1}^{3} \beta_{i}, \beta_{5}=1+\beta_{4}, \beta_{6}=2+\beta_{4}, \beta_{7}=\max \left(\beta_{5}\right.$, $\left.\beta_{6}\right), \beta_{8}=\sum_{i=1}^{3} b_{i}^{\prime}$.

Since $\left\|\vec{\psi}_{n}^{0}\right\|_{1} \leq b_{1}$, and $\left\|\vec{\psi}_{n}^{1}\right\|_{0} \leq b_{0}$, with $\beta_{9}=b_{0}+b_{1}+\beta_{7}$, then (35) is reduced to

$$
\left\|\vec{\psi}_{n t}(t)\right\|_{0}^{2}+\left\|\vec{\psi}_{n}(t)\right\|_{1}^{2} \leq \beta_{9}+\beta_{7} \int_{0}^{t}\left(\left\|\vec{\psi}_{n}\right\|_{0}^{2}+\left\|\vec{\psi}_{n t}\right\|_{1}^{2}\right) \mathrm{d} t .
$$

Appling the Belman-Gronwall (BGin) inequality, the abovementioned inequality gives $\forall t \in[0, T]$ :

$$
\begin{aligned}
\left\|\vec{\psi}_{n t}(t)\right\|_{0}^{2}+ & \left\|\vec{\psi}_{n}(t)\right\|_{1}^{2} \leq \beta_{9} e^{\beta_{7}}=b^{2}(c) \\
\Rightarrow & \left\|\vec{\psi}_{n t}(t)\right\|_{0}^{2} \leq b^{2}(c), \\
& \left\|\vec{\psi}_{n}(t)\right\|_{1}^{2} \leq b^{2}(c), \quad \forall t \in[0, T] .
\end{aligned}
$$

Easily, one can obtain that $\left\|\vec{\psi}_{n t}(t)\right\|_{Q} \leq b_{1}(c)$ and $\left\|\vec{\psi}_{n}(t)\right\|_{L^{2}(I, Y)} \leq b(c)$.

Then, by applying Alaoglu's theorem (Algth), $\left\{\vec{\psi}_{n}\right\}_{n \in N}$ has a subsequence; it is not loss of generality to say $\left\{\vec{\psi}_{n}\right\}_{n \in N}$ such that $\vec{\psi}_{n t} \rightarrow \vec{\psi}$ in $\left(L^{2}(\Pi)\right)^{3}$ and $\vec{\psi}_{n} \rightarrow \vec{\psi}$ in $\left(L^{2}(I, Y)\right)^{3}$, and

$$
\left(L^{2}(\mathscr{R}, Y)\right)^{3} \subset\left(L^{2}(\mathscr{R}, E)\right)^{3} \cong\left(\left(L^{2}(\mathscr{R}, E)\right)^{*}\right)^{3} \subset\left(L^{2}\left(\mathscr{R}, Y^{*}\right)\right)^{3} .
$$

Then, the Aubin compactness theorem [18] can be applied here to get that $\vec{\psi}_{n} \longrightarrow \vec{\psi}$ in $\left(L^{2}(\Pi)\right)^{3}$. Now, multiplying both sides of (27) and (29), and (31) by $\chi_{i}(t) \in C^{2}[0, T]$, such that $\chi_{i}(T)=\chi_{i}^{\prime}(T)=0, \chi_{i}(0) \neq 0$, $\chi_{i}^{\prime}(0) \neq 0, \forall i=1,2$, integrating on, finally integrating by parts twice the $1^{\text {st }}$ term of each one of the obtained three equations, led to

$$
\begin{aligned}
& -\int_{0}^{T} \frac{\mathrm{d}}{\mathrm{d} t}\left(\psi_{1 n}, v_{1 n}\right) \chi_{1}^{\prime}(t) \mathrm{d} t+\int_{0}^{T}\left[\left(\nabla \psi_{1 n}, \nabla v_{1 n}\right) \chi_{1}(t)+\left(\left(\psi_{1 n}, v_{1 n}\right)-\left(\psi_{2 n}, v_{1 n}\right)-\left(\psi_{3 n}, v_{1 n}\right)\right) \chi_{1}(t)\right] \mathrm{d} t \\
& =\int_{0}^{T}\left(k_{1}\left(\psi_{1 n}, \omega_{1}\right), v_{1 n}\right) \chi_{1}(t) \mathrm{d} t+\left(\psi_{1 n}^{1}, v_{1 n}\right) \chi_{1}(0), \\
& \quad \int_{0}^{T}\left(\psi_{1 n}, v_{1 n}\right) \chi_{1}^{\prime \prime}(t) \mathrm{d} t+\int_{0}^{T}\left[\left(\nabla \psi_{1 n}, \nabla v_{1 n}\right) \chi_{1}(t)+\left(\left(\psi_{1 n}, v_{1 n}\right)-\left(\psi_{2 n}, v_{1 n}\right)-\left(\psi_{3 n}, v_{1 n}\right)\right) \chi_{1}(t)\right] \mathrm{d} t \\
& =\int_{0}^{T}\left(k_{1}\left(\psi_{1 n}, \omega_{1}\right), v_{1 n}\right) \chi_{1}(t) \mathrm{d} t+\left(\psi_{1 n}^{1}, v_{1 n}\right) \chi_{1}(0)+\left(\psi_{1 n}^{0}, v_{1 n}\right) \chi_{1}^{\prime}(0), \\
& =\int_{0}^{T}\left(k_{2}\left(\psi_{2 n}, \omega_{2}\right), v_{2 n}\right) \chi_{2}(t) \mathrm{d} t+\left(\psi_{2 n}^{1}, v_{2 n}\right) \chi_{2}^{\prime}(t) \mathrm{d} t+\int_{0}^{T}\left[\left(\nabla \psi_{2 n}, \nabla v_{2 n}\right) \chi_{2}(t)+\left(\left(\psi_{2 n}, v_{2 n}\right)+\left(\psi_{3 n}, v_{2 n}\right)+\left(\psi_{1 n}, v_{2 n}\right)\right) \chi_{2}(t)\right] \mathrm{d} t \\
& \quad \int_{0}^{T}\left(\psi_{2 n}, v_{2 n}\right) \chi_{2}^{\prime \prime}(t) \mathrm{d} t+\int_{0}^{T}\left[\left(\nabla \psi_{2 n}, \nabla v_{2 n}\right) \chi_{2}(t)+\left(\left(\psi_{2 n}, v_{2 n}\right)+\left(\psi_{3 n}, v_{2 n}\right)+\left(\psi_{1 n}, v_{2 n}\right)\right) \chi_{2}(t) \mathrm{d} t\right] \\
& =\int_{0}^{T}\left(k_{3}\left(\psi_{3 n}, \omega_{3}\right), v_{3 n}\right) \chi_{3}(t) \mathrm{d} t+\left(\psi_{3 n}^{1}, v_{3 n}\right) \chi_{3}(0), \\
& \quad \frac{\mathrm{d} t}{\mathrm{~d} t}\left(\psi_{3 n}, v_{3 n}\right) \chi_{3}^{\prime}(t) \mathrm{d} t+\left[\int_{2 n}^{T}\left(\omega_{2}\right), v_{2 n}\right) \chi_{2}(t) \mathrm{d} t+\left(\psi_{2 n}^{1}, v_{2 n}\right) \chi_{2}(0)+\left(\psi_{2 n}^{0}, v_{2 n}\right) \chi_{2}^{\prime}(0), \\
& \left.\left.\quad \int_{3 n}\right) \chi_{3}(t)+\left(\left(\psi_{3 n}, v_{3 n}\right)+\left(\psi_{1 n}, v_{3 n}\right)-\left(\psi_{2 n}, v_{3 n}\right)\right) \chi_{3}(t) \mathrm{d} t\right]
\end{aligned}
$$




$$
\begin{gathered}
\int_{0}^{T}\left(\psi_{3 n}, v_{3 n}\right) \chi_{3}^{\prime \prime}(t) \mathrm{d} t+\int_{0}^{T}\left[\left(\nabla \psi_{3 n}, \nabla v_{3 n}\right) \chi_{3}(t)+\left(\left(\psi_{3 n}, v_{3 n}\right)+\left(\psi_{1 n}, v_{3 n}\right)-\left(\psi_{2 n}, v_{3 n}\right)\right) \chi_{3}(t) \mathrm{d} t\right] \\
=\int_{0}^{T}\left(k_{3}\left(\psi_{3 n}, \omega_{3}\right), v_{2 n}\right) \chi_{3}(t) \mathrm{d} t+\left(\psi_{3 n}^{1}, v_{3 n}\right) \chi_{3}(0)+\left(\psi_{3 n}^{0}, v_{3 n}\right) \chi_{3}^{\prime}(0) .
\end{gathered}
$$

Now, for each $i=1,2,3$, we have the following convergences:

First, since

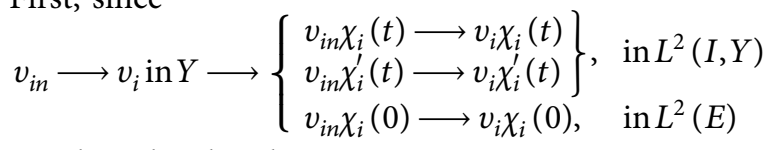

On the other hand, since

$$
\begin{aligned}
& \left.v_{\text {in }} \longrightarrow v_{i} \text { in } L^{2}(E)\right\} \longrightarrow \begin{cases}v_{\text {in }} \chi_{i}^{\prime}(t) \longrightarrow v_{i} \chi_{i}^{\prime}(t) \\
v_{\text {in }} \chi_{i}^{\prime \prime}(t) \longrightarrow v_{i} \chi_{i}^{\prime \prime}(t) \\
v_{\text {in }} \chi_{i}^{\prime}(0) \longrightarrow v_{i} \chi_{i}^{\prime}(0), \text { in } L^{2}(E)\end{cases} \\
& \text { Second, we have } \begin{cases}\psi_{\text {int }} \rightarrow \psi_{i t}, & \text { in } L^{2}(\Pi) \\
\psi_{\text {in }} \longrightarrow \psi_{i}, & \text { in } L^{2}(I, Y) \\
\psi_{\text {in }} \longrightarrow \psi_{i}, & \text { in } L^{2}(\Pi)\end{cases}
\end{aligned}
$$

Third, let $w_{\text {in }}=v_{\text {in }} \chi_{i}$ and $w_{i}=v_{i} \chi_{i}$, then $w_{\text {in }} \longrightarrow w_{i}$ in $L^{2}(\Pi)$ and $w_{\text {in }}$ is measurable in $E$, so from Assumption (A-I) and Proposition 1, the integral $\int_{\Pi} k_{i}\left(x, t, \psi_{i n}, \omega_{i}\right) w_{i n} \mathrm{~d} x \mathrm{~d} t$ is cont. with respect to $\left(\psi_{i n}, \omega_{i}, w_{i n}\right)$, then$$
\int_{0}^{T}\left(k_{i}\left(\psi_{i n}, \omega_{i}\right), v_{i n}\right) \chi_{i}(t) \mathrm{d} t \longrightarrow \int_{0}^{T}\left(k_{i}\left(\psi_{i}, \omega_{i}\right), v_{i}\right) \chi_{i}(t) \mathrm{d} t .
$$

Now, from these convergences and (24) and (25), for $(i=1)$, we can passage to the limits in (39) and (40) to get

$$
\begin{aligned}
& -\int_{0}^{T}\left(\psi_{1 t}, v_{1}\right) \chi_{1}^{\prime}(t) \mathrm{d} t+\int_{0}^{T}\left[\left(\nabla \psi_{1}, \nabla v_{1}\right)+\left(\psi_{1}-\psi_{2}-\psi_{3}, v_{1}\right)\right] \chi_{1}(t) \mathrm{d} t \\
& =\int_{0}^{T}\left(k_{1}\left(\psi_{1}, \omega_{1}\right), v_{1}\right) \chi_{1}(t) \mathrm{d} t+\left(\psi_{1}^{1}, v_{1}\right) \chi_{1}(0), \\
& \int_{0}^{T}\left(\psi_{1}, v_{1}\right) \chi_{1}^{\prime \prime}(t) \mathrm{d} t+\int_{0}^{T}\left[\left(\nabla \psi_{1}, \nabla v_{1}\right)+\left(\psi_{1}-\psi_{2}-\psi_{3}, v_{1}\right)\right] \chi_{1}(t) \mathrm{d} t \\
& =\int_{0}^{T}\left(k_{1}\left(\psi_{1}, \omega_{1}\right), v_{1}\right) \chi_{1}(t) \mathrm{d} t+\left(\psi_{1}^{1}, v_{1}\right) \chi_{1}(0)+\left(\psi_{1}^{0}, v_{1}\right) \chi_{1}^{\prime}(0),
\end{aligned}
$$

Case 1. Choose $\chi_{i} \in C^{2}[0, T]$, s.t. $\chi_{i}(0)=\chi_{i}^{\prime}(0)=\chi_{i}(T)=$ $\chi_{i}^{\prime}(T)=0$. Using these values in (47) (for $i=1$ ), using integration by parts twice for the first terms in the LHS of the obtained equation, yields to

$$
\begin{gathered}
\int_{0}^{T}\left\langle\psi_{1 t t}, v_{1}\right\rangle \chi_{1}(t) \mathrm{d} t+\int_{0}^{T}\left[\left(\nabla \psi_{1}, \nabla v_{1}\right)\right. \\
\left.+\left(\psi_{1}-\psi_{2}-\psi_{3}, v_{1}\right)\right] \chi_{1}(t) \mathrm{d} t \\
=\int_{0}^{T}\left(k_{1}\left(\psi_{1}, \omega_{1}\right), v_{1}\right) \chi_{1}(t) \mathrm{d} t
\end{gathered}
$$

which give that $\psi_{1}$ is a solution of (10) (a.e. on $I$ ).

Similar way can be used for $i=2,3$, with (41)-(44) respectively to get that $\psi_{2}$ and $\psi_{3}$ are solutions of and (12) and (14) respectively (a.e. on $I$ ).

Case 2. Choose $\chi_{i} \in C^{2}[0, T]$, such that $\chi_{i}(T) \neq 0$ and $\chi_{i}(0) \neq 0$. For $i=1$, integrating both sides of $(10)$ on $[0, T]$ after multiplying it by $\chi_{1}(t)$, using integrating by parts for the first term in the LHS of the obtained equation, then subtracting the obtained equation from (41), we get $\left(\psi_{i}^{1}, v_{i}\right) \chi_{i}(0)=\left(\psi_{i t}(0), v_{i}\right) \chi_{i}(0)$.

Also, similar way can be used but for $i=2,3$ with the pairs (12) and (47) and (14) and (48), respectively, to get the same result.

Case 3. Choose $\chi_{i} \in C^{2}[0, T]$, such that $\chi_{i}(0)=$ $\chi_{i}(T)=\chi_{i}^{\prime}(T)=0, \chi_{i}^{\prime}(0) \neq 0$. For $i=1$, integrating both sides of $(10)$ on $[0, T]$ after multiplying it by $\chi_{1}(t)$, using integrating by parts for the first term in the LHS of the obtained equation, then subtracting this obtained equation from (47), we get $\left(\psi_{i}^{0}, v_{i}\right) \chi_{i}^{\prime}(0)=\left(\psi_{i}(0), v_{i}\right) \chi_{i}^{\prime}(0)$. Also, for $i=2,3$ and by using (12) and (14), we can use a similar way to get the same result.

From the last two cases, easily we can get the ICs (11) and (13), and (15).

$\rightarrow$ Uniqueness of the solution: let $\vec{\psi}=\left(\psi_{1}, \psi_{2}, \psi_{3}\right)$ and $\vec{\psi}=\left(\bar{\psi}_{1}, \bar{\psi}_{2}, \bar{\psi}_{3}\right)$ be two solutions of the wkf (10)-(15), i.e., $\psi_{i}$ and $\bar{\psi}_{i}$ (for each $i=1,2,3$ ) are satisfied the wkf (10)-(15), subtracting each equality from the other and letting $v_{i}=\psi_{i}-\bar{\psi}_{i}$, yields to 


$$
\begin{aligned}
\left\langle\left(\psi_{i}-\psi_{i}\right)_{t t}, \psi_{i}-\bar{\psi}_{i}\right\rangle+\left\|\psi_{i}-\bar{\psi}_{i}\right\|_{1}^{2} & =\left(k_{i}\left(\psi_{i}, \omega_{i}\right)-k_{i}\left(\bar{\psi}_{i}, \omega_{i}\right), \psi_{i}-\bar{\psi}_{i}\right), \\
\left(\left(\psi_{i}-\bar{\psi}_{i}\right)(0), \psi_{i}-\bar{\psi}_{i}(0)\right) & =0 \\
\left(\left(\psi_{i}-\bar{\psi}_{i}\right)_{t}(0),\left(\psi_{i}-\bar{\psi}_{i}\right)_{t}(0)\right) & =0
\end{aligned}
$$

Adding these three equations, using Lemma 1.2 in ref. [18] on the first term in LHS of the obtained equation which will be positive, integrating both sides from 0 to $t$, using the initial conditions, the Lipshctiz property on the RHS, and lastly applying the B-G inequality, to get

$$
\begin{gathered}
\int_{0}^{t}\left[\frac{\mathrm{d}}{\mathrm{d} t}\left\|(\vec{\psi}-\vec{\psi})_{t}(t)\right\|_{0}^{2}+2\|(\vec{\psi}-\vec{\psi})\|_{1}^{2}\right] \mathrm{d} t \leq 2 L \int_{0}^{t}\left(\left\|(\vec{\psi}-\vec{\psi})_{t}(t)\right\|_{0}^{2}+\|(\vec{\psi}-\vec{\psi})\|_{1}^{2}\right) \mathrm{d} t \\
\left\|(\vec{\psi}-\vec{\psi})_{t}(t)\right\|_{0}^{2}+\|(\vec{\psi}-\overrightarrow{\bar{\psi}})\|_{1}^{2} \leq \Rightarrow\|(\vec{\psi}-\overrightarrow{\bar{\psi}})(t)\|_{1}^{2}=0, \quad \forall t \in I \Rightarrow \\
\|(\vec{\psi}-\vec{\psi})(t)\|_{\mathbf{L}^{2}(\mathbf{I}, Y)}=0 \Rightarrow, \quad \text { the solution is unique. }
\end{gathered}
$$

Lemma 1. In addition to Assumption $A$, if the functions $k_{i}$ (for each $i=1,2,3$ ) is Lip. with respect to $y_{i}$ and $\omega_{i}$, and if the control vector is bounded, then the operator $\vec{\omega} \mapsto \vec{\psi} \rightarrow \vec{\omega}$ from $\left(L^{2}(\Pi)\right)^{3}$ into $\left(L^{\infty}\left(I, L^{2}(E)\right)\right)^{3}$ or in to $\left(L^{2}(I, Y)\right)^{3} \stackrel{\omega}{\text { or }}$ in to $\left(L^{2}(\Pi)\right)^{3}$ is cont.

Proof. Let $\vec{\omega}=\left(\omega_{1}, \omega_{2}, \omega_{2}\right), \overrightarrow{\bar{\omega}}=\left(\bar{\omega}_{1}, \bar{\omega}_{2}, \bar{\omega}_{3}\right) \in\left(L^{2}(\Pi)\right)^{3}$, $\overrightarrow{\Delta \omega}=\vec{\omega}-\vec{\omega} \in\left(L_{\rightarrow}^{2}(\Pi)\right)_{\overrightarrow{3}}^{3}$, then by Theorem $1, \vec{\psi}=\vec{\psi} \vec{\omega}=$ $\left(\psi_{1}, \psi_{2}, \psi_{3}\right)$ and $\vec{\psi}=\vec{\psi} \underset{\omega}{\vec{\psi}}=\left(\bar{\psi}_{1}, \bar{\psi}_{2}, \bar{\psi}_{3}\right)$ are their corresponding states' solutions which satisfy the wkf of (10)-(15), setting $\overrightarrow{\Delta \psi}=\left(\Delta \psi_{1}, \Delta \psi_{2}, \Delta \psi_{3}\right)=\vec{\psi}-\vec{\psi}$, then

$$
\begin{gathered}
\left\langle\Delta \psi_{1 t t}, v_{1}\right\rangle+\left(\nabla \Delta \psi_{1}, \nabla v_{1}\right)+\left(\Delta \psi_{1}-\Delta \psi_{2}-\Delta \psi_{3}, v_{1}\right) \\
=\left(k_{1}\left(\psi_{1}+\Delta \psi_{1}, \omega_{1}+\Delta \omega_{1}\right)-k_{1}\left(\psi_{1}, \omega_{1}\right), v_{1}\right), \\
\Delta \psi_{1}(x, 0)=0, \\
\Delta \psi_{1 t}(x, 0)=0,
\end{gathered}
$$

$$
\begin{gathered}
\left\langle\Delta \psi_{2 t t}, v_{2}\right\rangle+\left(\nabla \Delta \psi_{2}, \nabla v_{2}\right)+\left(\Delta \psi_{2}+\Delta \psi_{1}+\Delta \psi_{3}, v_{2}\right) \\
=\left(k_{2}\left(\psi_{2}+\Delta \psi_{2}, \omega_{2}+\Delta \omega_{2}\right)-k_{2}\left(\psi_{2}, \omega_{2}\right), v_{2}\right), \\
\Delta \psi_{2}(x, 0)=0, \\
\Delta \psi_{2 t}(x, 0)=0, \\
\left\langle\Delta \psi_{3 t t}, v_{3}\right\rangle+\left(\nabla \Delta \psi_{3}, \nabla v_{3}\right)+\left(\Delta \psi_{3}+\Delta \psi_{1}-\Delta \psi_{2}, v_{3}\right) \\
=\left(k_{3}\left(\psi_{3}+\Delta \psi_{3}, \omega_{3}+\Delta \omega_{3}\right)-k_{3}\left(\psi_{3}, \omega_{3}\right), v_{3}\right), \\
\Delta \psi_{3}(x, 0)=0, \\
\Delta \psi_{3 t}(x, 0)=0 .
\end{gathered}
$$

Substituting $v_{i}=\Delta \psi_{i t}$ for each $i=1,2,3$ in (51), (53), and (55), respectively, adding the obtained three equations together, and using the same way that we used to get (32), a similar equation can be obtained but with $\overrightarrow{\Delta \psi}$ in state of $\vec{\psi}_{n}$, and then integration of both sides on $[0, t]$, using the Lip. property on $k_{i}, i=1,2,3$, with respect to each dependent variable, yields

$$
\begin{aligned}
\int_{0}^{t} \frac{\mathrm{d}}{\mathrm{d} t}\left[\left\|\overrightarrow{\Delta \psi}_{t}(t)\right\|_{0}^{2}+\|\overrightarrow{\Delta \psi}\|_{1}^{2}\right] \leq & 2 \int_{0}^{t}\left[\left(\left|\Delta \psi_{2}\right|+\left|\Delta \psi_{3}\right|\right)\left|\Delta \psi_{1 t}\right|+\left(\left|\Delta \psi_{1}\right|+\left|\Delta \psi_{3}\right|\right)\left|\Delta \psi_{2 t}\right|\right] \mathrm{d} t \\
& +2 \int_{0}^{t}\left[\left(\left|\Delta \psi_{1}\right|+\left|\Delta \psi_{2}\right|\right)\left|\Delta \psi_{3 t}\right|+\left(\bar{L}_{1}\left|\Delta \psi_{1}\right|+\overline{\bar{L}}_{1}\left|\Delta \omega_{1}\right|\right)\left|\Delta \psi_{1 t}\right|\right] \mathrm{d} t \\
& +2 \int_{0}^{t}\left[\bar{L}_{2}\left(\left|\Delta \psi_{2}\right|+\overline{\bar{L}}_{2}\left|\Delta \omega_{2}\right|\right)\left|\Delta \psi_{2 t}\right|+\bar{L}_{3}\left(\left|\Delta \psi_{3}\right|+\overline{\bar{L}}_{3}\left|\Delta \omega_{3}\right|\right)\left|\Delta \psi_{3 t}\right|\right] \mathrm{d} t .
\end{aligned}
$$


Using the definitions of the norms and the relations between them, we get

$$
\begin{aligned}
\left\|\overrightarrow{\Delta \psi}_{t}(t)\right\|_{0}^{2}+\|\overrightarrow{\Delta \psi}\|_{1}^{2} \leq & 2 \int_{0}^{t}\left(\|\overrightarrow{\Delta \psi}\|_{0}^{2}+\left\|\overrightarrow{\Delta \psi_{t}}\right\|_{1}^{2}\right) \mathrm{d} t+\widetilde{L}_{1} \int_{0}^{t}\left(\|\overrightarrow{\Delta \psi}\|_{0}^{2}+\left\|\overrightarrow{\Delta \psi_{t}}\right\|_{1}^{2}\right) \mathrm{d} t \\
& +\bar{L}^{2} \int_{0}^{T}\|\overrightarrow{\Delta \omega}\|_{0}^{2} \mathrm{~d} t+\bar{L}^{2} \int_{0}^{t}\left\|\overrightarrow{\Delta \psi_{t}}\right\|_{1}^{2} \mathrm{~d} t \\
\leq & \bar{L}^{2}\|\overrightarrow{\Delta \omega}(t)\|_{\Pi}^{2}+L_{1} \int_{0}^{t}\left(\|\overrightarrow{\Delta \psi}\|_{0}^{2}+\left\|\overrightarrow{\Delta \psi_{t}}\right\|_{1}^{2}\right) \mathrm{d} t
\end{aligned}
$$

where $\tilde{L}_{1}=\max \left(\bar{L}_{1}, \bar{L}_{2}, \bar{L}_{3}\right), \quad \bar{L}^{2}=\max \left(\overline{\bar{L}}_{1}, \overline{\bar{L}}_{2}, \overline{\bar{L}}_{3}\right)$, and Applying the BGin, with $L^{2}=\bar{L}^{2} e^{L_{1}}$, we get $L_{1}=\max \left(2+\widetilde{L}_{1}, 2+\widetilde{L}_{1}+\bar{L}^{2}\right)$.

$$
\begin{aligned}
\left\|\overrightarrow{\Delta \psi}_{t}(t)\right\|_{0}^{2}+\|\overrightarrow{\Delta \psi}\|_{1}^{2} & \leq L^{2}\|\overrightarrow{\Delta \omega}(t)\|_{\Pi}^{2}, \quad \forall t \in \bar{I} \Rightarrow \\
\|\overrightarrow{\Delta \psi}(t)\|_{1}^{2} & \leq L^{2}\|\overrightarrow{\Delta \omega}(t)\|_{\Pi}^{2}, \quad \forall t \in \bar{I} \Rightarrow \\
\|\overrightarrow{\Delta \psi}\|_{L^{\infty}\left(I, L^{2}(\mathrm{E})\right)} & \leq L\|\overrightarrow{\Delta \omega}\|_{\Pi}, \\
\|\overrightarrow{\Delta \psi}\|_{L^{2}(I, Y)} & \leq L\|\overrightarrow{\Delta \omega}\|_{\Pi} \text { and }\|\overrightarrow{\Delta \psi}\|_{\Pi} \leq L\|\overrightarrow{\Delta \omega}\|_{\Pi} .
\end{aligned}
$$

From the abovementioned three inequalities, the Lip. continuity of the operator $\vec{\omega} \mapsto \vec{\psi}$ easily obtained.

1.3. The Existence of a Classical Optimal Control. This section concerned with proving the existence theorem with a CCOPCV satisfying the EQVC and INEQVC is studied.
Hence, the following assumption and lemmas will be needed.

Assumption B. Consider $m_{r i}$ (for $r=0, \ldots, q$ and $i=1,2,3$ ) is of Caraty. on $\Pi \times\left(\mathscr{R} \times \complement_{i}\right)$ and satisfies $\psi_{i} \in \mathscr{R}$ and $\omega_{i} \in \mathcal{C}_{i}$ :

$$
\left|m_{r i}\left(x, t, \psi_{i}, \omega_{i}\right)\right| \leq M_{r i}(x, t)+c_{k i} \psi_{i}^{2}, \quad \text { where } M_{r i} \in L^{1}(\Pi), \forall i=1,2,3, \forall r=0, \ldots, q \text {. }
$$

Lemma 2. With Assumption $B$, the functional $\vec{\omega} \mapsto M_{r}(\vec{\omega})$ is cont. on $\left(L^{2}(\Pi)\right)^{3}$.

Proof. Using Assumption B and Proposition 1 gives $\int_{Q} m_{r i}\left(x, t, \psi_{i}, \omega_{i}\right) \mathrm{d} x \mathrm{~d} t$ is cont. on $L^{2}(\Pi), \forall i=1,2,3$, and $\forall r=0, \ldots, q$; hence, $M_{l}(\vec{\omega})$ is cont. on $\left(L^{2}(\Pi)\right)^{3}$.

Lemma 3 (see [13]). Let $m: Q \times \mathscr{R}^{2} \longrightarrow \mathscr{R}$ is of Caraty. on $\Pi \times(\mathscr{R} \times \mathscr{R})$ and satisfies $|m(x, \psi, \omega)| \leq m(x, t)+c \psi^{2}$, where $(x, t) \in L^{1}(\Pi), \omega \in \mathcal{C}, c \geq 0, \mathcal{C} \subset \mathscr{R}$ is compact. Then, $\int_{\Pi} m(x, \psi, \omega) d x$ is cont. on $L^{2}(\Pi)$, with respect to $\psi$.

Theorem 2. With the Assumptions $A$ and $B$, if the set $\vec{C}$ is convex (cox.) and compact (com), $\vec{W}_{A} \neq \varnothing$, and the function $k_{i}(\forall i=1,2,3)$ has the form

$$
k_{i}\left(x, t, \psi_{i}, \omega_{i}\right)=k_{i 1}\left(x, t, \psi_{i}\right)+k_{i 2}(x, t) \omega_{i},
$$

where $\quad\left|k_{i 1}\left(x, t, \psi_{i}\right)\right| \leq \eta_{i}(x, t)+c_{i}\left|\psi_{i}\right|, \quad\left|k_{i 2}(x, t)\right| \leq k_{i}$, $\eta_{i} \in L^{2}(\Pi), c_{i} \geq 0$.
In addition $m_{r i}$ is independent of $\omega_{i}(\forall i=1,2,3$, and $r=1, \ldots, p), m_{r i}(\forall i=1,2,3$, and $r=1, \ldots, p)$ is cox. with respect to $\omega_{i}$ for fixed $\left(x, t, \psi_{i}\right)$, there exists a CCOPCV.

Proof. From the Assumption on $C_{i} \subset \mathscr{R} \forall i=1,2,3$ and Egorov's theorem, one obtains that $\vec{W}_{1} \times \vec{W}_{2} \times \vec{W}_{3}=\overrightarrow{\widehat{W}}$ is weakly com. Since $\vec{W}_{A} \neq \varnothing$, hence there is $\vec{\omega} \in \vec{W}_{A}$ s.t. $r(\overrightarrow{\vec{\omega}})=0,1 \leq r \leq p, M_{k}(\overrightarrow{\vec{\omega}}) \leq 0$, for $p+1 \leq r \leq q$ and there is a minimizing sequence $\left\{\vec{\omega}_{\rho}\right\}$ s.t. $\vec{\omega}_{\rho} \in \overrightarrow{\widehat{W}}_{A}, \forall \rho$, which satisfies $\quad \rho \stackrel{\lim }{\longrightarrow} \infty M_{0}\left(\vec{\omega}_{\rho}\right)=\overrightarrow{\bar{\omega}} \in \vec{W}_{A} M_{0}(\overrightarrow{\bar{\omega}})$. Since $\vec{\omega}_{\rho} \in \vec{W}_{A}, \forall \rho$ and $\vec{W}$ is weakly com., then $\left\{\vec{\omega}_{\rho}\right\}$ has a subsequence say again $\left\{\vec{\omega}_{\rho}\right\}$ which converges weakly to some $\vec{\omega}$ in $\vec{W}$, i.e., $\vec{\omega}_{\rho}-\omega$ in $\left(L^{2}(\Pi)\right)^{3}$ and $\left\|\vec{\omega}_{\rho}\right\|_{\Pi} \leq c, \forall \rho$. From Theorem 1 , for any given control $\vec{\omega}_{\rho}$, then $\vec{\psi}_{\rho}=\vec{\psi}_{\vec{\omega}_{\rho}}$ is a unique solution for the Tsteqs, and $\|\vec{\psi}\|_{\rho L^{2}(I, Y)}$, 
$\|\vec{\psi}\|_{\rho t L^{2}(\Pi)}$ are bounded, then by Algth, the sequences $\left\{\vec{\psi}_{\rho}\right\}$ and $\left\{\vec{\psi}_{\rho t}\right\}$ have a weakly converging subsequences, say for simplicity, $\left\{\vec{\psi}_{\rho}\right\}$ and $\left\{\vec{\psi}_{\rho t}\right\}$, i.e.,

$$
\begin{gathered}
\vec{\psi}_{\rho} \rightarrow \vec{\psi}, \quad \text { in }\left(L^{2}(I, Y)\right)^{3}, \\
\vec{\psi}_{\rho t} \rightarrow \vec{\psi}_{t}, \quad \text { in }\left(L^{2}(\Pi)\right)^{3} .
\end{gathered}
$$

Then, by applying the Aubin compactness theorem [18], the sequence $\left\{\vec{\psi}_{\rho}\right\}$ has a strongly converging subsequence, say for simplicity, $\left\{\vec{\psi}_{\rho}\right\}$ such that $\vec{\psi}_{\rho} \longrightarrow \vec{\psi}$ in $\left(L^{2}(\Pi)\right)^{3}$.

Now, for each $\rho$, substitute the solution $\left(\psi_{1 \rho}, \psi_{2 \rho}, \psi_{3 \rho}\right)$ in the wkf of (18), (20), and (22), then multiply both sides of each one by $\chi_{i}(t)$ (with $\chi_{i} \in C^{2}[0, T]$, such that $\chi_{i}(T)=\chi_{i}^{\prime}(T)=0, \chi_{i}(0) \neq 0, \chi_{i}^{\prime}(0) \neq 0$, for $\left.i=1,2,3\right)$. After rewriting the first terms in the LHS in each one of them, integrating both sides on $[0, T]$, and then by applying integration by parts for these first terms, we get

$$
\begin{aligned}
& \int_{0}^{T} \frac{\mathrm{d}}{\mathrm{d} t}\left(\psi_{1 \rho t}, v_{1}\right) \chi_{1}(t) \mathrm{d} t+\int_{0}^{T}\left[\left(\nabla \psi_{1 \rho}, \nabla v_{1}\right)+\left(\psi_{1 \rho}, v_{1}\right)-\left(\psi_{2 \rho}, v_{1}\right)-\left(\psi_{3 \rho}, v_{1}\right)\right] \chi_{1}(t) \mathrm{d} t \\
& \quad=\int_{0}^{T}\left(k_{11}\left(x, t, \psi_{1 \rho}\right)+k_{12}(x, t) \omega_{1 \rho}, v\right) \chi_{1}(t) \mathrm{d} t, \\
& \int_{0}^{T} \frac{\mathrm{d}}{\mathrm{d} t}\left(\psi_{2 \rho t}, v_{2}\right) \chi_{2}(t) \mathrm{d} t+\int_{0}^{T}\left[\left(\nabla \psi_{2 \rho}, \nabla v_{2}\right)+\left(\psi_{2 \rho}, v_{2}\right)+\left(\psi_{1 \rho}, v_{2}\right)+\left(\psi_{3 \rho}, v_{2}\right)\right] \chi_{2}(t) \mathrm{d} t \\
& \quad=\int_{0}^{T}\left(k_{21}\left(x, t, \psi_{2 \rho}\right)+k_{22}(x, t) \omega_{2 \rho}, v_{2}\right) \chi_{2}(t) \mathrm{d} t, \\
& \int_{0}^{T} \frac{\mathrm{d}}{\mathrm{d} t}\left(\psi_{3 \rho t}, v_{3}\right) \chi_{3}(t) \mathrm{d} t+\int_{0}^{T}\left[\left(\nabla \psi_{3 \rho}, \nabla v_{3}\right)+\left(\psi_{3 \rho}, v_{3}\right)+\left(\psi_{1 \rho}, v_{3}\right)-\left(\psi_{2 \rho}, v_{3}\right)\right] \chi_{3}(t) \mathrm{d} t \\
& =\int_{0}^{T}\left[\left(k_{31}\left(x, t, \psi_{3 \rho}\right)+k_{32}(x, t) \omega_{3 \rho}, v_{3}\right) \chi_{3}(t)\right] \mathrm{d} t .
\end{aligned}
$$

One can passage the limits in the LHS of (50)-(52) by applying the same manner which is applied in the proof of Theorem 1 to passage the limits in RHS of these equations; we suppose $(\forall i=1,2,3), \quad v_{i} \in C[\overline{\mathrm{E}}], \quad w_{i}=v_{i} \chi_{i}(t)$, then $w_{i} \in C[\bar{\Pi}] \in L^{\infty}(I, V) \subset L^{2}(\Pi)$, set $\bar{k}_{i 1}\left(\psi_{1 \rho}\right)=k_{i 1}\left(\psi_{i \rho}\right) w_{i}$, then $\bar{k}_{i 1}: \Pi \times \mathbb{R} \longrightarrow \mathbb{R}$ is of Caraty., using Proposition 1 , to get the integral $\int_{\Pi} k_{i 1}\left(\psi_{i \rho}\right) w_{i} \mathrm{~d} x \mathrm{~d} t$ is cont. with respect to $\psi_{i \rho}$, but $\psi_{i \rho} \longrightarrow \psi_{i}$ in $L^{2}(\Pi)$ and $\omega_{i \rho} \rightarrow \omega_{i}$ in $L^{2}(\Pi)$, then

$$
\begin{gathered}
\int_{\Pi} k_{i 1}\left(\psi_{i \rho}\right) w_{i} \mathrm{~d} x \mathrm{~d} t \longrightarrow \int_{\Pi} k_{i 1}\left(\psi_{i}\right) w_{i} \mathrm{~d} x \mathrm{~d} t, \quad \forall w_{i} \in C[\bar{\Pi}] \text { for } i=1,2,3, \\
\int_{\Pi} k_{i 2}(x, t) \omega_{i \rho} w_{i} \mathrm{~d} x \mathrm{~d} t \longrightarrow \int_{\Pi} k_{i 2}(x, t) \omega_{i} w_{i} \mathrm{~d} x \mathrm{~d} t, \quad \forall w_{i} \in C[\bar{\Pi}], \text { for } i=1,2,3 .
\end{gathered}
$$

Thus, (66) and (67) are holded for every $v_{i} \in \Upsilon$, since $C(\bar{E})$ is dense in $\Upsilon$; hence, we get the wkf (10), (12), and (14).

Also, the same manner which is applied in the proof of Theorem 2 can be used here to passage the limits in the ICs. Hence, $\left(\psi_{1}, \psi_{2}, \psi_{3}\right)$ is a solution of the wkf of (10)-(15).

On the other hand, since $m_{r i}$ (for $i=1,2,3$ and $r=1,2, \ldots, p)$ is independent of $\omega_{i}$ and cont. with respect to $\psi_{i}$, by Lemma $2, \int_{\Pi} m_{r i}\left(x, t, \psi_{i \rho}\right) \mathrm{d} x \mathrm{~d} t$ is cont. with respect to $y_{i}, \vec{\psi}_{\rho} \longrightarrow \vec{\psi}$ in $\left(L^{2}(\Pi)\right)^{3}$, then

$\int_{\Pi} m_{r i}\left(x, t, \psi_{i \rho}\right) \mathrm{d} x \mathrm{~d} t \longrightarrow \int_{\Pi} m_{r i}\left(x, t, \psi_{i}\right) \mathrm{d} x \mathrm{~d} t$.

Hence, $M_{r}(\vec{\omega})=\rho \stackrel{\lim }{\longrightarrow} \infty M_{r}\left(\vec{\omega}_{\rho}\right)=0$.
Now, since $m_{r i}\left(x, t, \psi_{i}, \omega_{i}\right) \quad$ (for each $r=0,1+$ $p, 2+p, \ldots, q$ and $i=1,2,3)$ is cont. with respect to $\left(\psi_{i}, \omega_{i}\right)$ and since $C_{i}$ is compact, then using Lemma 3, we get

$$
\int_{\Pi} m_{r i}\left(x, t, \psi_{i \rho}, \omega_{i \rho}\right) \mathrm{d} x \mathrm{~d} t \longrightarrow \int_{\Pi} m_{r i}\left(x, t, \psi_{i}, \omega_{i \rho}\right) \mathrm{d} x \mathrm{~d} t .
$$

On the other hand, $\int_{\Pi} m_{r i}\left(x, t, \psi_{i}, \omega_{i \rho}\right) \mathrm{d} x \mathrm{~d} t$ is cox. and cont. with respect to $\omega_{i}$ (since $m_{r i}\left(x, t, \psi_{i}, \omega_{i}\right)$ is cox. and cont. with respect to $\left.\omega_{i}\right)$, then $\int_{\Pi} m_{r i}\left(x, t, \psi_{i}, \omega_{i}\right) \mathrm{d} x \mathrm{~d} t$ Necessary and Sufficient is weakly lower semicont. (welsc) with respect to $\omega_{i}$, i.e., 


$$
\begin{aligned}
& \int_{\Pi} m_{r i}\left(x, t, \psi_{i}, \omega_{i}\right) \mathrm{d} x \mathrm{~d} t \leq \rho \stackrel{\lim }{\longrightarrow} \infty i n f \int_{\Pi}\left(m_{r i}\left(x, t, \psi_{i}, \omega_{i \rho}\right)-m_{r i}\left(x, t, \psi_{i \rho}, \omega_{i \rho}\right)\right) \mathrm{d} x \mathrm{~d} t \\
&+\rho \stackrel{\lim }{\longrightarrow} \infty \inf \int_{\Pi} m_{r i}\left(x, t, \psi_{i \rho}, \omega_{i \rho}\right) \mathrm{d} x \mathrm{~d} t \leq \rho \stackrel{\lim }{\longrightarrow} \infty i n f \int_{\Pi} m_{r i}\left(x, t, \psi_{i \rho}, \omega_{i \rho}\right) \mathrm{d} x \mathrm{~d} t, \quad \text { by }(55) \Rightarrow \\
& \sum_{i=1}^{2} \int_{\Pi} m_{r i}\left(x, t, \psi_{i}, \omega_{i}\right) \mathrm{d} x \mathrm{~d} t \leq k \stackrel{\lim }{\longrightarrow} \infty i n f \sum_{i=1}^{2} \int_{\Pi} m_{r i}\left(x, t, \psi_{i k}, \omega_{i \rho}\right) \mathrm{d} x \mathrm{~d} t \Rightarrow \\
& M_{r}(\vec{\omega}) \leq \lim _{\rho \rightarrow \infty} \inf M_{r}\left(\vec{\omega}_{\rho}\right),
\end{aligned}
$$

then $M_{r}(\vec{\omega}) \leq 0(r=p+1, \ldots, q)$ since $\vec{\omega}_{\rho} \in \overrightarrow{\widehat{W}}_{A}, \forall \rho$, and we get that

$$
\begin{aligned}
M_{0}(\vec{\omega}) & \leq \rho \stackrel{\lim }{\longrightarrow} \infty \inf M_{0}\left(\vec{\omega}_{\rho}\right) \\
& =\lim _{\rho \longrightarrow \infty} M_{0}\left(\vec{\omega}_{\rho}\right)=\overrightarrow{\bar{\omega}} \in \vec{W}_{A} M_{0}(\overrightarrow{\bar{\omega}}) \\
& =\vec{\omega} \in \vec{W}_{A} M_{0}(\overrightarrow{\bar{\omega}}) .
\end{aligned}
$$

Thus, $\vec{\omega}$ is a CCOPCV.

Assumption C. Assume for $r=0, \ldots, q$ and $i=1,2,3$, the functions $k_{i}, k_{i \psi_{i}}, k_{i \omega_{i}}, m_{r_{i} \psi_{i}}$, and $m_{r_{i} \omega_{i}}$ are defined and are of
Caraty. on $\Pi \times\left(\mathscr{R} \times \complement^{\prime}\right)$ (where $C^{\prime}$ is an open subset in $\complement$ ) and satisfy $\left|k_{i \psi_{i}}\left(x, t, \psi_{i}, \omega_{i}\right)\right| \leq L_{i},\left|k_{i \omega_{i}}\left(x, t, \psi_{i}, \omega_{i}\right)\right| \leq L_{i}^{\prime}$ :

$$
\begin{aligned}
& \left|m_{r_{i} \psi_{i}}\left(x, t, \psi_{i}, \omega_{i}\right)\right| \leq M_{r_{i 5}}(x, t)+c_{r_{i 5}}\left|\psi_{i}\right|, \\
& \left|m_{r_{i} \omega_{i}}\left(x, t, \psi_{i}, \omega_{i}\right)\right| \leq M_{r_{i 6}}(x, t)+c_{r_{i 6}}\left|\psi_{i}\right|,
\end{aligned}
$$

where $\quad(x, t) \in \Pi, \quad \psi_{i}, \omega_{i} \in \mathbb{R}, \quad M_{r_{i 5}}, M_{r_{i 6}} \in L^{2}(\Pi)$. $L_{i}, L_{i}^{\prime}, c_{r_{i 5}}, c_{r_{i 6}} \geq 0$.

Note: for simplicity, in the following theorem, we will drop the index $k$ from the functions $m_{l i}$ and $M_{l}$. Also, we assume the Assums. (A), (B), and (C) are considered.

Theorem 3. Consider the TAEqs $\vec{\xi}=\left(\xi_{1}, \xi_{2}, \xi_{3}\right)$ of Tseqs (1)-(6) are defined by

$$
\begin{aligned}
\xi_{1 t t}-\Delta \xi_{1}+\xi_{1}+\xi_{2}+\xi_{3} & =\xi_{1} k_{1 \psi_{1}}\left(x, t, \psi_{1}, \omega_{1}\right)+m_{1 \psi_{1}}\left(x, t, \psi_{1}, \omega_{1}\right), \quad \text { on } \Pi, \\
\xi_{1} & =0, \quad \text { on } \Sigma \quad \xi_{1}(x, T)=\xi_{1 t}(x, T)=0, \quad \text { on } E, \\
\xi_{2 t t}-\Delta \xi_{2}+\xi_{2}-\xi_{1}-\xi_{3} & =\xi_{2} k_{2 \psi_{2}}\left(x, t, \psi_{2}, \omega_{2}\right)+m_{2 \psi_{2}}\left(x, t, \psi_{2}, \omega_{2}\right), \quad \text { on } \Pi, \\
\xi_{2} & =0, \quad \text { on } \Sigma, \quad \xi_{2}(x, T)=\xi_{2 t}(x, T)=0, \quad \text { on } E, \\
\xi_{3 t t}-\Delta \xi_{3}+\xi_{3}-\xi_{1}+\xi_{2} & =\xi_{13} k_{3 \psi_{3}}\left(x, t, \psi_{3}, \omega_{3}\right)+m_{3 \psi_{3}}\left(x, t, \psi_{3}, \omega_{3}\right), \quad \text { on } \Pi, \\
\xi_{3} & =0, \quad \text { on } \Sigma \quad \xi_{3}(x, T)=\xi_{3 t}(x, T)=0, \quad \text { on } E,
\end{aligned}
$$

and the Ham is given by $\mathscr{H}(x, t, \vec{\psi}, \vec{\omega}, \vec{\xi})=$ $\sum_{i=1}^{3} \zeta_{i}\left(x, t, \psi_{i}, \omega_{i}\right), \quad$ where $\quad \zeta_{i}=\xi_{i} k_{i}\left(x, t, \psi_{i}, \omega_{i}\right)+$ $m_{i}\left(x, t, \psi_{i}, \omega_{i}\right)$, for each $i=1,2,3$.

Then, the Frde. of $G$ is defined by

$$
M^{\prime}(\vec{\omega}) \Delta \vec{\omega}=\int_{\Pi} \mathscr{H} \underset{\omega}{ }(x, t, \vec{\psi}, \vec{\omega}, \vec{\xi}) \Delta \vec{\omega} \mathrm{d} x \mathrm{~d} t
$$

where $\quad \mathscr{H}_{\vec{\omega}}(x, t, \vec{\psi}, \vec{\xi}, \vec{\omega})=\left(\zeta_{1 \omega_{1}}, \zeta_{1 \omega_{1}}, \zeta_{1 \omega_{1}}\right)$, and $\Delta \vec{\omega}=\left(\Delta \omega_{1}, \Delta \omega_{2}, \Delta \omega_{3}\right)$,

with $\zeta_{i \omega_{i}}=\xi_{i} k_{i \omega_{i}}\left(x, t, \psi_{i}, \omega_{i}\right)+m_{i \omega_{i}}\left(x, t, \psi_{i}, \omega_{i}\right), \quad m_{i}=\sum_{r=0}^{q} \kappa_{r} m_{r i}$ and $\xi_{i}=\sum_{r=0}^{q} \kappa_{r} \xi_{l i}$, for each $i=1,2,3$.

Proof. at first, let the wkf of the TAEqs are given $\forall v_{1}, v_{2}, v_{3} \in Y$, by

$$
\begin{aligned}
& \left\langle\xi_{1 t t}, v_{1}\right\rangle+\left(\nabla \xi_{1}, \nabla v_{1}\right)+\left(\xi_{1}+\xi_{2}+\xi_{3}, v_{1}\right)=\left(\xi_{1} k_{1 \psi_{1}}+m_{1 \psi_{1}}, v_{1}\right), \quad \forall v_{1} \in Y \text { a.e. on } I \\
& \left(\xi_{1}(T), v_{1}\right)=\left(\xi_{1 t}(T), v_{1}\right)=0 \\
& \left\langle z \xi_{2 t}, v_{2}\right\rangle+\left(\nabla \xi_{2}, \nabla v_{2}\right)+\left(\xi_{2}-\xi_{1}-\xi_{3}, v_{2}\right)=\left(\xi_{2} k_{2 \psi_{2}}+m_{2 \psi_{2}}, v_{2}\right), \quad \forall v_{2} \in Y \text { a.e. on } I \\
& \left(\xi_{2}(T), v_{2}\right)=\left(\xi_{2 t}(T), v_{2}\right)=0 \\
& \left\langle\xi_{3 t}, v_{3}\right\rangle+\left(\nabla \xi_{3}, \nabla v_{3}\right)+\left(\xi_{3}-\xi_{1}+\xi_{2}, v_{3}\right)=\left(\xi_{3} k_{3 \psi_{3}}+m_{3 \psi_{3}}, v_{3}\right), \quad \forall v_{2} \in Y \text { a.e. on } I \\
& \left(\xi_{3}(T), v_{3}\right)=\left(\xi_{3 t}(T), v_{3}\right)=0
\end{aligned}
$$


From the assumptions and using the same manner which is applied in the proof of Theorem 1, once can prove that the wkf (75)-(80) has a unique solution $\vec{\xi}=\left(\xi_{1}, \xi_{2}, \xi_{3}\right) \in\left(L^{2}(\Pi)\right)^{3}$.
Substituting $v_{i}=\Delta \psi_{i}$ for each $i=1,2,3$ in (75), (77), and (79) and integrating both sides on $[0, T]$, we get

$$
\begin{aligned}
& \int_{0}^{T}\left[\left\langle\Delta \psi_{1}, \xi_{1 t t}\right\rangle+\left(\nabla \xi_{1}, \nabla \Delta \psi_{1}\right)+\left(\xi_{1}+\xi_{3}+\xi_{2}, \Delta \psi_{1}\right)\right] \mathrm{d} t=\int_{0}^{T}\left(\xi_{1} k_{1 \psi_{1}}+g_{1 \psi_{1}}, \Delta \psi_{1}\right) \mathrm{d} t \\
& \int_{0}^{T}\left[\left\langle\Delta \psi_{2}, \xi_{2 t t}\right\rangle+\left(\nabla \xi_{2}, \nabla \Delta \psi_{2}\right)+\left(\xi_{2}-\xi_{1}-\xi_{3}, \Delta \psi_{2}\right)\right] \mathrm{d} t=\int_{0}^{T}\left(\xi_{2} k_{2 \psi_{2}}+g_{2 \psi_{2}}, \Delta \psi_{2}\right) \mathrm{d} t \\
& \int_{0}^{T}\left[\left\langle\Delta \psi_{3}, \xi_{3 t t}\right\rangle+\left(\nabla \xi_{3}, \nabla \Delta \psi_{3}\right)+\left(\xi_{3}-\xi_{1}+\xi_{2}, \Delta \psi_{3}\right)\right] \mathrm{d} t=\int_{0}^{T}\left(\xi_{3} k_{3 \psi_{3}}+g_{3 \psi_{3}}, \Delta \psi_{3}\right) \mathrm{d} t .
\end{aligned}
$$

Now, let $\vec{\omega}, \vec{\omega} \in\left(L^{2}(\Pi)\right)^{3}, \overrightarrow{\Delta \omega}=\overrightarrow{\vec{\omega}}-\vec{\omega} \in\left(L^{2}(Q)\right)^{3}$, and then by Theorem $1, \vec{\psi}=\vec{\psi} \vec{\omega} \underset{\longrightarrow}{\longrightarrow}$ and $\vec{\psi}=\vec{\psi} \underset{\vec{\omega}}{\vec{\psi}}$ are their corresponding solutions. Set $\overrightarrow{\Delta \psi}=\left(\Delta \psi_{1}, \Delta \psi_{2}, \Delta \psi_{3}\right)=$ $\vec{\psi}-\vec{\psi}$, substitute $v_{i}=\xi_{i}$ for each $i=1,2,3$ in (51), (53), and (55), integrate both sides on $[0, T]$, and then integrate by parts twice the first term in the LHS of each equation. Finding for each $i=1,2,3$ the Frde. of $k_{i}$ in the RHS of each equation which are exist from the Assumption $\mathrm{C}$, then by Lemma 1, and the inequality of Minkowiski, one has

$$
\begin{gathered}
\int_{0}^{T}\left[\left\langle\Delta \psi_{1}, \xi_{1 t t}\right\rangle+\left(\nabla \Delta \psi_{1}, \nabla \xi_{1}\right)+\left(\Delta \psi_{1}-\Delta \psi_{2}-\Delta \psi_{3}, \xi_{1}\right)\right] \mathrm{d} t \\
\quad=\int_{0}^{T}\left(k_{1 \psi_{1}} \Delta \psi_{1}+k_{1 \omega_{1}} \Delta \omega_{1}, \xi_{1}\right) \mathrm{d} t+O_{1}(\overrightarrow{\Delta \omega})\|\overrightarrow{\Delta \omega}\|_{\Pi}, \\
\int_{0}^{T}\left[\left\langle\Delta \psi_{2}, \xi_{2 t t}\right\rangle+\left(\nabla \Delta \psi_{2}, \nabla \xi_{2}\right)+\left(\Delta \psi_{2}+\Delta \psi_{1}+\Delta \psi_{3}, \xi_{2}\right) \mathrm{d} t\right] \\
\quad=\int_{0}^{T}\left(k_{2 \psi_{2}} \delta \psi_{2}+k_{2 \omega_{2}} \Delta \omega_{2}, \xi_{2}\right) \mathrm{d} t+O_{2}(\overrightarrow{\Delta \omega})\|\overrightarrow{\Delta \omega}\|_{\Pi}, \\
\int_{0}^{T}\left[\left\langle\Delta \psi_{3}, \xi_{3 t t}\right\rangle+\left(\nabla \delta \psi_{3}, \nabla \xi_{3}\right)+\left(\Delta \psi_{2 \varepsilon}+\Delta \psi_{1}-\Delta \psi_{2}, \xi_{3}\right)\right] \mathrm{d} t \\
\quad=\int_{0}^{T}\left(k_{3 \psi_{3}} \Delta \psi_{3}+k_{3 \omega_{3}} \Delta \omega_{3}, \xi_{3}\right) \mathrm{d} t+O_{3}(\overrightarrow{\Delta \omega})\|\overrightarrow{\Delta \omega}\|_{\Pi} .
\end{gathered}
$$

Subtracting (84)-(86) from, respectively, adding the obtain equations, we get

$$
\int_{0}^{T} \sum_{i=1}^{3}\left(k_{i \omega_{i}} \Delta \omega_{i}, \xi_{i}\right) \mathrm{d} t+O_{4}(\overrightarrow{\Delta \omega})=\int_{0}^{T} \sum_{i=1}^{3}\left(m_{i \psi_{i}}, \Delta \psi_{i}\right) \mathrm{d} t
$$

where. $\rightarrow \longrightarrow O_{4}(\varepsilon) \longrightarrow 0$, as $\overrightarrow{\Delta \omega} \longrightarrow 0$, with $O_{4}(\overrightarrow{\Delta \omega})=$ $\sum_{i=1}^{3} O_{i}(\overrightarrow{\Delta \omega}) \overrightarrow{\Delta \omega}_{\Pi}$

On the other hand, from the Assumption B on $m_{i}$ (for $i=1,2,3)$, the Frde definition, Lemma 1, and by applying the inequality of Minkowiski, we obtain

$$
\begin{aligned}
M(\vec{\omega}+\overrightarrow{\Delta \omega})-M(\vec{\omega})= & \int_{\Pi} \sum_{i=1}^{3}\left(m_{i \psi_{i}} \Delta \psi_{i}+m_{i \omega_{i}} \Delta \omega_{i}\right) \mathrm{d} x \mathrm{~d} t \\
& +O_{5}(\overrightarrow{\Delta \omega}) \overrightarrow{\Delta \omega}_{\Pi},
\end{aligned}
$$

where $O_{5}(\overrightarrow{\Delta \omega}) \longrightarrow 0$, as $\overrightarrow{\Delta \omega} \longrightarrow 0$.

Now, by substituting (87) in (88), one has

$$
\begin{aligned}
M(\vec{\omega}+\overrightarrow{\Delta \omega})-M(\vec{\omega})= & \int_{\Pi} \sum_{i=1}^{3} \zeta_{i \omega_{i}}\left(x, t, \psi_{i}, \omega_{i}\right) \Delta \omega_{i} \mathrm{~d} x \mathrm{~d} t \\
& +O_{6}(\overrightarrow{\Delta \omega}) \overrightarrow{\Delta \omega}_{\Pi},
\end{aligned}
$$

where $O_{6}(\overrightarrow{\Delta \omega})=O_{5}(\overrightarrow{\Delta \omega})+O_{5}(\overrightarrow{\Delta \omega}) \longrightarrow 0$, as $\overrightarrow{\Delta \omega} \longrightarrow 0$.

Finally, from the Frde. of, we get

$$
M^{\prime}(\vec{\omega}) \Delta \vec{\omega}=\int_{\Pi} \mathscr{C}_{\vec{\omega}}(x, t, \vec{\psi}, \vec{\omega}, \vec{\xi}) \Delta \vec{\omega} \mathrm{d} x \mathrm{~d} t
$$

1.4. Necessary and Sufficient Conditions for Optimality. This section deals with the theorems for the Necoop necessary under certain hypotheses which are proved as follows: 
Theorem 4. Necoop (multipliers theorem):

(a) with Assumptions $A, B$, and $C$, if $\overrightarrow{\widehat{W}}$ is cox. and the $\vec{\omega} \in \vec{W}_{A}$ is optimal, then there are multipliers $\kappa_{r} \in \mathbb{R}, r=0,1, \ldots, p, p+1, \ldots, q$ with $\kappa_{r} \geq 0$, for $r=0, p+1, \ldots, q, \sum_{r=0}^{q}\left|\kappa_{r}\right|=1$ such that the following Kuhn-Tucker-Lagrange (K. T. L.) conditions are holded:

$$
\begin{gathered}
\int_{\Pi} \mathscr{H}_{\vec{\omega}}(x, t, \vec{\psi}, \vec{\omega}, \vec{\xi}) \Delta \vec{\omega} \mathrm{d} x \mathrm{~d} t \geq 0, \quad \forall \vec{\omega} \in \overrightarrow{\widehat{W}}, \overrightarrow{\Delta \omega}=\vec{\omega}-\vec{\omega}, \\
\kappa_{r} M_{r}(\vec{\omega})=0, \quad \text { for } r=p+1, \ldots, q .
\end{gathered}
$$

(b) Inequality (91) is equivalent to the (weak) piecewise minimum principle

$$
\mathscr{H}_{\vec{\omega}}(x, t, \vec{\psi}, \vec{\xi}, \vec{\omega}) \cdot \vec{\omega}(t)=\min _{\bar{\omega} \longrightarrow \epsilon \vec{c}} \mathscr{H}_{\vec{\omega}}(x, t, \vec{\psi}, \vec{\xi}, \vec{\omega}) \cdot \overrightarrow{\bar{\omega}}(t), \quad \text { a.e. on } \Pi \text {. }
$$

Proof (a) From Lemma 2, the functional $M_{r}(\vec{\omega})$ (for $r=0,1, \ldots, q)$ is cont., and from Theorem 3, the functional $M_{r}^{\prime}$ (for $r=0,1, \ldots, q$ ) is cont. with respect to $\overrightarrow{\bar{\omega}}-\vec{\omega}$ and linear in $\vec{\omega}-\vec{\omega}$, then $M_{k}^{\prime}$ is $L$-differential for every $L$, and then applying the K. T. L. theorem [5], there are multipliers $\kappa_{r} \in \mathbb{R}, r=0,1, \ldots, q$ with $\kappa_{r} \geq 0$, for $r=0, p+1, \ldots, q, \sum_{r=0}^{q}\left|\kappa_{r}\right|=1$, such that (91)-(93) are satisfied, by using Theorem 3, then (91) becomes $\quad \sum_{r=0}^{q} \kappa_{r} \int_{\Pi} \sum_{i=1}^{3} \zeta_{i \omega_{i}}$ $\left(x, t, \psi_{i}, \omega_{i}\right) \Delta \omega_{i} \mathrm{~d} x \mathrm{~d} t \geq 0$, which can be rewritten as

$$
\begin{aligned}
\int_{\Pi} \vec{\zeta}_{\vec{\omega}} \cdot\left(\overrightarrow{\bar{\omega}}_{k}-\vec{\omega}\right) \mathrm{d} x \mathrm{~d} t & \geq 0, \quad \forall \overrightarrow{\bar{\omega}} \in \overrightarrow{\widehat{W}} \text { with } \vec{\zeta} \vec{\omega} \\
& =\left(\zeta_{1 \omega_{1}}, \zeta_{2 \omega_{2}}, \zeta_{3 \omega_{3}}\right) .
\end{aligned}
$$

(b) Let $\left\{\vec{\omega}_{l}\right\}$ be a dense sequence (dse) in $\overrightarrow{\widehat{W}}, m$ denotes the Lebesgue measure on $\Pi$, and $\Gamma \subset \Pi$ be a measurable subset with property $\overrightarrow{\bar{\omega}}(x, t)=\left\{\begin{array}{l}\overrightarrow{\bar{\omega}}_{l}(x, t), \text { if }(x, t) \in \Gamma \\ \vec{\omega}(x, t), \text { if }(x, t) \notin \Gamma\end{array}\right.$.

Therefore, (94) becomes

$$
\begin{array}{r}
\int_{\Gamma} \vec{\zeta}_{\vec{\omega}} \cdot(\overrightarrow{\bar{\omega}}-\vec{\omega}) \mathrm{d} x \mathrm{~d} t \geq 0 \text {, which implies to } \vec{\zeta} \vec{\omega} \\
\cdot\left(\overrightarrow{\bar{\omega}}_{l}-\vec{\omega}\right) \geq 0 \text {, a.e. on } \prod
\end{array}
$$

This means the inequality is satisfied on the whole region $Q$ except in a subset $\Pi_{l}$ such that $m\left(\Pi_{l}\right)=0, \forall l$, where $m$ represents the Lebesgue measure; thus, the inequality holds on $\Pi$ except in the union $\cup_{l} \Pi_{l}$ with $m\left(\cup_{l} \Pi_{l}\right)=0$, but $\left\{\overrightarrow{\bar{\omega}}_{l}\right\}$ is a dse in $\vec{W}$, then there is $\vec{\omega} \in \vec{W}$ such that $\int_{\Pi} \vec{\zeta} \vec{\omega} \cdot\left(\overrightarrow{\bar{\omega}}_{l}-\vec{\omega}\right) \geq 0$, a.e. on $\Pi, \forall \overrightarrow{\bar{\omega}} \in \overrightarrow{\widehat{W}}$.

That is, (91) gives (94). The converse is clear.

Theorem 5. Sucoop: besides the Assumptions A, B, and $C$, suppose $\overrightarrow{\widehat{W}}$ is cox., with $\vec{C}$ cox., $k_{i}$ and $m_{r i}(\forall r=1,2, \ldots, p)$ are affine with respect to $\left(\psi_{i}, \omega_{i}\right)$ for each $(x, t), m_{r i}(\forall r=$ $0, p+1, \ldots, q)$ are cox. with respect to $\left(\psi_{i}, \omega_{i}\right) \forall(x, t)$, for $i=1,2,3$. Then, the Necoop for Theorem 4 with $\kappa_{0}>0$ are sufficient.

Proof. Assume $\vec{\omega} \in \vec{W}_{A}$ satisfies the condition (91) and (92). Let $M(\vec{\omega})=\sum_{r=0}^{q} \kappa_{r} M_{r}(\vec{\omega})$, then using Theorem 3, we get

$$
M^{\prime}(\vec{\omega}) \Delta \vec{\omega}=\sum_{r=0}^{q} \kappa_{r} M_{r}^{\prime}(\vec{\omega}) \Delta \vec{\omega}=\sum_{r=0}^{q} \kappa_{r} \int_{\Pi} \sum_{i=1}^{3} \zeta_{i \omega_{i}} \cdot \delta \omega_{i} \mathrm{~d} x \mathrm{~d} t \geq 0
$$

Since

$$
k_{i}\left(x, t, \psi_{i}, \omega_{i}\right)=
$$

$k_{i 1}(x, t) \psi_{i}+k_{i 2}(x, t) \omega_{i}+k_{i 3}(x, t), i=1,2,3$.

Let $\omega=\left(\omega_{1}, \omega_{2}, \omega_{3}\right)$ and $\overrightarrow{\bar{\omega}}=\left(\bar{\omega}_{1}, \bar{\omega}_{2}, \bar{\omega}_{3}\right)$ be two given control vectors, then $\vec{\psi}=\left(\psi_{\omega 1}, \psi_{\omega 2}, \psi_{\omega 3}\right)=\left(\psi_{1}, \psi_{2}, \psi_{3}\right)$ and $\overrightarrow{\bar{\psi}}=\left(\bar{\psi}_{\omega 1}, \bar{\psi}_{\bar{\omega} 2}, \bar{\psi}_{\bar{\omega} 3}\right)=\left(\bar{\psi}_{1}, \bar{\psi}_{2}, \bar{\psi}_{3}\right)$ represent the corresponding stats solutions. Substituting $(\vec{\omega}, \vec{y})$ in (1)-(6), multiplying all the obtained equalities by $\alpha \in[0,1]$ once and on the other hand substituting $(\vec{w}, \vec{y})$ in (1)-(6), multiplying all the obtained equalities by $(1-\alpha)$ once again, and lastly adding each pair from the corresponding equalities together, we obtain 


$$
\begin{aligned}
& \left(\alpha \psi_{1}+(1-\alpha) \bar{\psi}_{1}\right)_{t t}-\Delta\left(\alpha \psi_{1}+(1-\alpha) \bar{\psi}_{1}\right)+\alpha\left(\psi_{1}-\psi_{2}-\psi_{3}\right)+(1-\alpha)\left(\bar{\psi}_{1}-\bar{\psi}_{2}-\bar{\psi}_{3}\right) \\
& =\alpha\left(k_{11}(x, t) \psi_{1}+k_{12}(x, t) \omega_{1}\right)+(1-\alpha)\left(k_{11}(x, t) \bar{\psi}_{1}+k_{12}(x, t) \bar{\omega}_{1}\right)+k_{13}(x, t), \\
& \alpha \psi_{1}(x, t)+(1-\alpha) \bar{\psi}_{1}(x, 0)=0, \\
& \alpha \psi_{1}(x, 0)+(1-\alpha) \bar{\psi}_{1}(x, 0)=\psi_{1}^{0}(x) \\
& \alpha \psi_{1 t}(x, 0)+(1-\alpha) \bar{\psi}_{1 t}(x, 0)=\psi_{1}^{1}(x) \text {, } \\
& \left(\alpha \psi_{2}+(1-\alpha) \bar{\psi}_{2}\right)_{t t}-\Delta\left(\alpha \psi_{2}+(1-\alpha) \bar{\psi}_{2}\right)+\alpha\left(\psi_{2}+\psi_{1}+\psi_{3}\right)+(1-\alpha)\left(\bar{\psi}_{2}+\bar{\psi}_{1}+\bar{\psi}_{3}\right)=\alpha \\
& \left(k_{21}(x, t) \psi_{2}+k_{22}(x, t) \omega_{2}\right)+(1-\alpha)\left(k_{21}(x, t) \bar{\psi}_{2}+k_{22}(x, t) \bar{\omega}_{2}\right)+k_{23}(x, t) \text {, } \\
& \alpha \psi_{2}(x, t)+(1-\alpha) \bar{\psi}_{2}(x, 0)=0, \\
& \alpha \psi_{2}(x, 0)+(1-\alpha) \bar{\psi}_{2}(x, 0)=\psi_{2}^{0}(x), \\
& \alpha \psi_{2 t}(x, 0)+(1-\alpha) \bar{\psi}_{2}(x, 0)=\psi_{2}^{1}(x) \text {, } \\
& \left(\alpha \psi_{3}+(1-\alpha) \bar{\psi}_{3}\right)_{t t}-\Delta\left(\alpha \psi_{3}+(1-\alpha) \bar{\psi}_{3}\right)+\alpha\left(\psi_{3}+\psi_{1}-\psi_{2}\right)+(1-\alpha)\left(\bar{\psi}_{3}+\bar{\psi}_{1}-\bar{\psi}_{2}\right) \\
& =\alpha\left(k_{31}(x, t) \psi_{3}+k_{32}(x, t) \omega_{3}\right)+(1-\alpha)\left(k_{31}(x, t) \bar{\psi}_{3}+k_{32}(x, t) \bar{\omega}_{3}\right)+k_{23}(x, t), \\
& \alpha \psi_{3}(x, t)+(1-\alpha) \bar{\psi}_{3}(x, 0)=0, \\
& \alpha \psi_{3}(x, 0)+(1-\alpha) \bar{\psi}_{3}(x, 0)=\psi_{3}^{0}(x), \\
& \alpha \psi_{3 t}(x, 0)+(1-\alpha) \bar{\psi}_{3 t}(x, 0)=\psi_{3}^{1}(x) \text {. }
\end{aligned}
$$

Equations (97)-(105) show that if the control vector is $\overrightarrow{\widetilde{\omega}}=\left(\widetilde{\omega}_{1}, \widetilde{\omega}_{2}, \widetilde{\omega}_{3}\right)$ with $\overrightarrow{\widetilde{\omega}}=\vec{\omega}+(1-\alpha) \overrightarrow{\bar{\omega}}$ then its corresponding state vector is $\overrightarrow{\widetilde{\psi}}=\left(\widetilde{\psi}_{1}, \widetilde{\psi}_{2}, \widetilde{\psi}_{3}\right)$ with $\widetilde{\psi}_{i}=\psi_{i \tilde{\omega}_{i}}=\psi_{i\left(\alpha \omega_{i}+(1-\alpha) \bar{\omega}_{i}\right)}=\alpha \psi_{i}+(1-\alpha) \bar{\psi}_{i}, \forall i=1,2,3$.

This gives the operator $\vec{\omega} \mapsto \vec{y} \underset{\omega}{\longrightarrow}$ is convex-linear with respect to $(\vec{\psi}, \vec{\omega})$ for any $(x, t) \stackrel{\omega}{\epsilon} \Pi$.

On the other hand, the function $M_{l}(\vec{\omega})$ (for $k=1, \ldots, p)$ is convex-linear with respect to $(\vec{\psi}, \vec{\omega})$ for any $(x, t) \in \Pi$, this backs to the fact that the sum of two affine functions $m_{r i}\left(x, t, \psi_{i}, \omega_{i}\right)$ (for each $i=1,2,3$, and $(r=1, \ldots, p)$ with respect to $\left(\psi_{i}, \omega_{i}\right)$ and $\forall(x, t) \in \Pi$ is affine and the operator $\vec{\omega} \mapsto \vec{\psi} \underset{\omega}{ }$ is convex-linear.

The functions $M_{r}(\vec{\omega}), \forall r=0, p+1, \ldots, q$ are cox. with respect to $(\vec{\psi}, \vec{\omega}), \forall(x, t) \in \Pi$ (from the assumptions on the functions $m_{r 1}$ and $\left.m_{r 2}, \forall r=0, p+1, \ldots, q\right)$. Hence, $M(\vec{\omega})$ is cox. with respect to $(\vec{\psi}, \vec{\omega}), \forall(x, t) \in \Pi$ in the cox. set $\overrightarrow{\widehat{W}}$, and has a cont. Fréd satisfies $M^{\prime}(\vec{\omega}) \cdot(\overrightarrow{\bar{\omega}}-\vec{\omega}) \geq 0 \Rightarrow M(\vec{\omega})$ has a minimum at $\vec{\omega} \Rightarrow M(\vec{\omega}) \leq M(\vec{\omega}), \forall \vec{\omega} \in \overrightarrow{\widehat{W}} \rightrightarrows$

$$
\sum_{r=0}^{p} \kappa_{r} M_{r}(\vec{\omega})+\sum_{r=p+1}^{q} \kappa_{r} M_{r}(\vec{\omega}) \leq \sum_{r=0}^{p} \kappa_{r} M_{r}(\overrightarrow{\bar{\omega}})+\sum_{r=p+1}^{q} \kappa_{r} M_{r}(\overrightarrow{\bar{\omega}}), \quad \forall \vec{\omega} \in \overrightarrow{\widehat{W}}
$$

Let $\overrightarrow{\bar{\omega}} \in \overrightarrow{\widehat{W}}_{A}$, with $k_{k} \geq 0$ (for $r=p+1, \ldots, q$ ), then from (8), (9), and (77), the abovementioned inequality becomes

$$
\kappa_{0} M_{0}(\vec{\omega}) \leq \kappa_{0} M_{0}(\vec{\omega}), \quad \forall \vec{\omega} \in \overrightarrow{\widehat{W}} \Rightarrow G_{0}(\vec{\omega}) \leq G_{0}(\vec{\omega}), \quad \forall \vec{\omega} \in \overrightarrow{\widehat{W}} \Rightarrow . \therefore \vec{\omega} \text { is a CCOCVE. }
$$

\section{Conclusions}

The Galm with the Aubin compactness theorem are applied successfully to prove the existence of a unique "continuous state vector" solution for the TNLHPDEqs for a given cont. CCOPCVE. The existence theorem of governing by the considered the TNLHPDEqs with EQVC and INEQVC is proved. The existence of a unique solution of ATEqs associated with the considered Tsteqs is studied. The Frde. of the Ham is derived. The theorems of the 
Necoop and the Sucoop of the constrained problem are proved.

\section{Data Availability}

No data were used to support this study.

\section{Conflicts of Interest}

The authors declare that they have no conflicts of interest.

\section{Acknowledgments}

The authors would like to extend their sincere thanks to Mustansiriyah University for providing a pleasurable and stimulating environment to carry their research. Also, they wish to express their sincere esteems to College of Science, Department of Mathematics, for their timely support.

\section{References}

[1] D. L. Andrea, "Optimal control and treatment of infectious diseases. The case of huge treatment costs," Mathematics, vol. 4, no. 21, pp. 1-27, 2016.

[2] D. La Torre, H. Kunze, F. Mendivil, M. Ruiz Galan, and R. Zaki, "Inverse problems: theory and application to science and engineering 2015," Mathematical Problems in Engineering, vol. 2015, no. 2, Article ID 890527, 3 pages, 2015.

[3] J. Kahuru, S. L. Livingstone, and Y. Nkansah-Gyekye, "Optimal control techniques on a mathematical model for the dynamics of tungiasis in a community," International Journal of Mathematics and Mathematical Sciences, vol. 2017, no. 19, pp. 1-19, 2015.

[4] E. Trélat, J. Zhu, and E. Zuazua, "Allee optimal control of a system in ecology," Mathematical Models and Methods in Applied Sciences, vol. 28, no. 9, pp. 1665-1697, 2018.

[5] Y. O. Aderinto, A. O. Afolabi, and I. T. Issa, "On optimal planning of electric power generation systems," Journal of Mathematics, vol. 50, no. 1, pp. 89-95, 2018.

[6] J. Zhu and E. T. M. Cerf, "Geometric optimal control and applications to aerospace," Journal of Mathematics for Industry, vol. 9, no. 8, 2017.

[7] J. Warga, Optimal Control of Differential and Functional Equations, Academic Press, New York, NY, USA, 1972.

[8] J. L. Lions, Optimal Control of Systems Governed by Partial Differential Equations, Springer, New York, NY, USA, 1972.

[9] D. Bors and S. Walczak, "Optimal control elliptic systems with distributed and boundary controls," Nonlinear Analysis: Theory, Methods \& Applications, vol. 63, no. 5-7, pp. e1367-e1376, 2005.

[10] J. Al-Hawasy, "The continuous classical optimal control of a nonlinear hyperbolic equation (CCOCP)," Al-Mustansiriyah Journal of Science, vol. 19, no. 8, pp. 96-110, 2008.

[11] I. Chryssoverghi and J. Al-Hawasy, "The continuous classical optimal control problem of a semilinear parabolic equations," Journal of KerbalaUniversity, vol. 8, no. 3, pp. 57-70, 2010.

[12] J. Al-Hawasy and E. Al-Rawdhanee, "The continuous classical optimal control of a coupled of non-linear elliptic equations," Mathematical Theory and Modeling, vol. 4, no. 14, pp. 211-219, 2014.

[13] J. A. A. Al-Hawasy and G. M. Kadhem, "The continuous classical optimal control for a coupled nonlinear parabolic partial differential equations with equality and inequality constraints," Journal of Al-Nahrain University-Science, vol. 19, no. 1, pp. 173-186, 2016.

[14] J. Kadhem, "The continuous classical optimal control of a couple nonlinear hyperbolic partial differential equations with equality and inequality constraints," Iraqi Journal for Science, vol. 57, no. 2C, pp. 1528-1538, 2016.

[15] J. Al-Hawasy and D. K. Jasim, "The continuous classical optimal control problems of a triple elliptic partial differential equations," Ibn Al-Haitham for Pure and Applied Science, vol. 33, no. 1, pp. 143-151, 2020.

[16] J. Al-Hawasy and M. A. Jaber, "The continuous classical optimal control governing by triple parabolic boundary value problem," Ibn Al-Haitham for Pure and Applied Science, vol. 33, no. 1, pp. 129-142, 2020.

[17] I. Chyssoverghi, Optimization, National Technical University of Athens, Athens, Greece, 2003.

[18] R. Temam, Navier-Stokes Equations, North-Holand Publishing Company, Amsterdam, Netherlands, 1977. 